\title{
Majorana quasiparticles in semiconducting carbon nanotubes
}

\author{
Magdalena Marganska, ${ }^{1}$ Lars Milz, ${ }^{1}$ Wataru Izumida,,${ }^{1,2}$ Christoph Strunk, ${ }^{3}$ and Milena Grifoni ${ }^{1, *}$ \\ ${ }^{1}$ Institute for Theoretical Physics, University of Regensburg, 93053 Regensburg, Germany \\ ${ }^{2}$ Department of Physics, Tohoku University, Sendai 980 8578, Japan \\ ${ }^{3}$ Institute for Experimental and Applied Physics, University of Regensburg, 93053 Regensburg, Germany
}

(Received 10 November 2017; published 22 February 2018)

\begin{abstract}
Engineering effective $p$-wave superconductors hosting Majorana quasiparticles (MQPs) is nowadays of particular interest, also in view of the possible utilization of MQPs in fault-tolerant topological quantum computation. In quasi-one-dimensional systems, the parameter space for topological superconductivity is significantly reduced by the coupling between transverse modes. Together with the requirement of achieving the topological phase under experimentally feasible conditions, this strongly restricts in practice the choice of systems which can host MQPs. Here, we demonstrate that semiconducting carbon nanotubes (CNTs) in proximity with ultrathin $s$-wave superconductors, e.g., exfoliated $\mathrm{NbSe}_{2}$, satisfy these needs. By precise numerical tight-binding calculations in the real space, we show the emergence of localized zero-energy states at the CNT ends above a critical value of the applied magnetic field, of which we show the spatial evolution. Knowing the microscopic wave functions, we unequivocally demonstrate the Majorana nature of the localized states. An effective four-band model in the $k$-space, with parameters determined from the numerical spectrum, is used to calculate the topological phase diagram and its phase boundaries in analytic form. Finally, the impact of symmetry breaking contributions, like disorder and an axial component of the magnetic field, is investigated.
\end{abstract}

DOI: 10.1103/PhysRevB.97.075141

\section{INTRODUCTION}

Majorana fermions, particles being their own antiparticle predicted already eighty years ago [1], have remained elusive to experimental observation so far. Hence recent proposals to observe quasiparticles with the Majorana property-the socalled Majorana quasiparticles (MQPs)—in one-dimensional (1D) hybrid systems containing superconducting elements [2] have raised big attention. The most popular implementations are based on semiconducting nanowires with large spin-orbit interaction and large $g$-factor, proximity coupled to a conventional superconductor $[3,4]$. When a magnetic field is applied to the nanowire in the direction perpendicular to the effective spin-orbit field, a topologically nontrivial phase is expected when the induced Zeeman splitting is large enough to overcome the superconducting gap. Signatures of MQP behavior include, e.g., a quantized zero-bias peak emerging in transport spectra while sweeping the magnetic field. Setups with epitaxially grown superconductor-semiconducting nanowires are by now the most advanced experimentally, and the emergence of a zero bias transport peak at finite magnetic field has been reported by various groups [5-8].

Zero-bias peaks can, however, also emerge due to the coalescence of Andreev bound states [8,9]—naturally occurring in confined normal conductor-superconductor systemsor due to the development of Kondo correlations [10]. An unambiguous theoretical confirmation of the experimental observation of MQPs would require an accurate microscopic modeling of the nanowires. However, diameters of many tens

*Corresponding author: milena.grifoni@ur.de of nanometers and lengths of several micrometers hinder truly microscopic calculations of the electronic spectrum of finite systems. The real space models of semiconductor nanowires are usually constructed in a top-down approach, starting with an effective model and quantizing it on a chosen crystal lattice [11]. Without accurate modeling of experimental setups, one can make only qualitative, rather than quantitative predictions of the boundaries of the topological phase. Recently, MQP signatures have also been observed in Kitaev chains of magnetic adatoms on superconducting substrates $[12,13]$. The microscopic modeling of ferromagnetic chains is, however, still in development $[14,15]$. In this work, we consider carbon nanotubes (CNTs) as host for MQPs. Due to their small diameter, they can be considered as truly $1 \mathrm{D}$ conductors with one relevant transverse mode for each valley and spin. The low-energy spectrum of the CNTs is well described in terms of tight-binding models for carbon atoms on a rolled graphene lattice [16]. Experimental advances in the preparation of ultraclean CNTs have allowed to measure their transport spectra in various transport regimes [17], and hence to gain confidence in the accuracy of the theoretical modeling. Two proposals to observe MQPs in carbon nanotubes have been based on spiral magnetic fields [18], induced, e.g., by magnetic domains [19], or on large electrical fields [20]. Despite their appeal due to the possibility of inducing large extrinsic spinorbit coupling, these setups are quite sophisticated and either hard to realize experimentally or to model microscopically.

The setup, which we describe here is, similar to Ref. [21], based solely on the intrinsic curvature-induced spin-orbit coupling of CNTs. The physical setup is shown in Fig. 1(a) and consists of a CNT placed on an ultrathin superconducting film, with a gating layer beneath and the magnetic field applied 
(a)

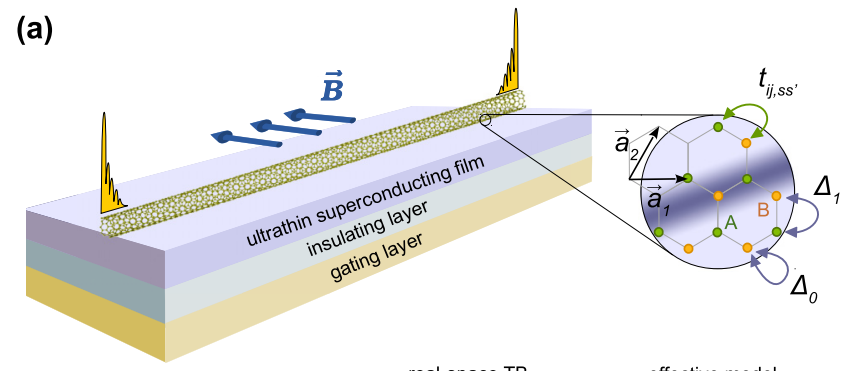

(b)
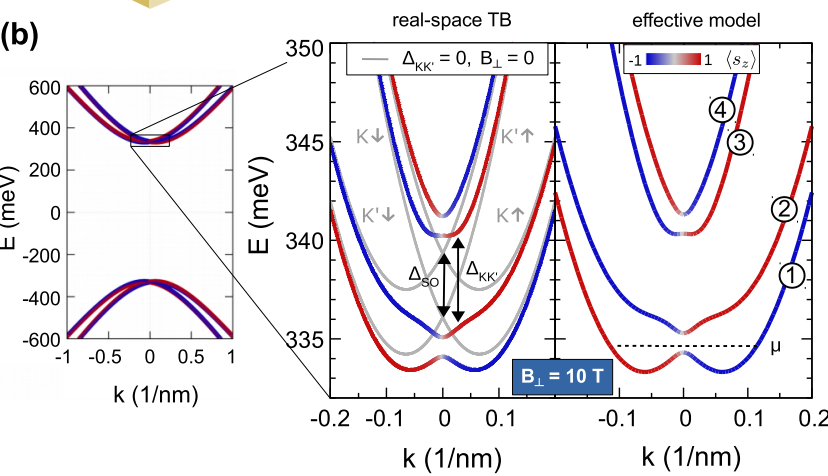

FIG. 1. Setup and bulk properties of a proximitized nanotube. (a) Schematic of the system, the CNT with its proximal superconductor and a gating layer. A magnetic field is applied in parallel to the substrate and perpendicular to the nanotube. We find Majorana quasiparticles at the ends of the CNT/superconductor hybrid. The ingredients of our model are shown in the inset. The nearest-neighbor hopping $t_{i j, s s^{\prime}}$ is spin-dependent because of spin-orbit coupling. The superconducting substrate (i) breaks the rotational symmetry of the nanotube, as shown by the darker strip with finite electrostatic on-site potential, and (ii) induces superconducting pairing in the nanotube, with on-site $\left(\Delta_{0}\right)$ and nearest-neighbor $\left(\Delta_{1}\right)$ pairing correlations. (b) The energy bands of a $(12,4)$ nanotube in the vicinity of the Dirac points are shown in the leftmost plot, with red/blue corresponding to spin up/down (quantized along the nanotube axis) bands. Our region of interest here is the neighborhood of the $\Gamma$ point in the conduction band. The enlarged plots show the spectrum in this region, obtained both in the real-space tight-binding calculation and in an analytical effective model. The spin-orbit splitting between the Kramers doublets at $k=0, B_{\perp}=0$ is $\Delta_{\text {SO }}$ (here equal $2 \mathrm{meV}$ ), and the width of the anticrossing opening between different valley states is $\Delta_{K K^{\prime}}$ (here $2.5 \mathrm{meV}$ ). Grey lines shown in the plot correspond to subbands without the valley mixing. There we can assign spin and valley quantum number to each band. With the valley mixing, $B_{\perp}$ is able to open a gap at $k=0$.

parallel to the film and perpendicular to the nanotube. The subband degeneracies at $k=0$ are broken by the valley mixing, caused by the interaction with the substrate, and a magnetic field applied perpendicular to the CNT axis. In contrast to [21], we consider semiconducting rather than metallic CNTs, since the Fermi velocity in the former is lower by a factor of $\sim 10^{-3}$ than in the latter. Because the Fermi velocity controls the localization properties of Majorana bound states, semiconducting CNTs can host Majorana end states at a thousand times smaller length than the metallic ones. As we shall show, MQPs indeed arise at the end of proximitized CNTs with a length of only a few micrometers (instead of millimeters in the case of a metallic CNT), easily handled in the device synthesis.
The core of Ref. [21] is an effective four-band model including valley mixing, a perpendicular magnetic field and superconducting pairing terms. By analyzing the Pfaffian topological invariant, they show that without mixing the valleys, the Bogoliubov-de Gennes spectrum is always gapless, and show by a numerical calculation that the BdG spectrum of these systems contains zero-energy states in the region where the Pfaffian invariant is nontrivial. Hence they provide the first indication that the proposed setup can indeed host MQPs. Unlike in Ref. [21], the starting point for our investigation is a microscopic, tight-binding model of the CNT lattice, with external influences such as the substrate potential, superconducting pairings, magnetic field (perpendicular $B_{\perp}$ and axial $B_{\|}$) or disorder added in the real space. We find numerically, using sparse matrix algorithms, the energetically lowest Bogoliubov-de Gennes levels and their corresponding wave functions, for an infinitely long as well as for a finite $(6 \mu \mathrm{m}$ long) $(12,4)$ CNT. The emergence of localized zero-energy states above a critical magnetic field strength is demonstrated in the real space. Using the knowledge of the components of the wave functions, we prove the Majorana nature of the localized states. Furthermore, the full three-dimensional spatial profile of the Majorana bound states is provided.

While the microscopic approach allows a more accurate description of the proximitized CNT, effective models in the $k$ space are useful for analytical estimates of the topological phase diagram and give a better understanding of the underlying physics. Thus, in our work, we construct an effective Hamiltonian in the reciprocal space which well reproduces the numerically calculated low-energy spectrum and its evolution across the topological phase transition. The effective model allows us to gain the knowledge of the system's symmetries and topological invariants. We have performed a full analysis of our system's symmetries, revealing its membership of the BDI instead of the D symmetry class. We discuss the relationship between the Pfaffian invariant (natural in class $D$ and used, e.g., in Ref. [21]) and the winding number topological invariant appropriate for class BDI, and identify the presence of two different topologically nontrivial phases in the $\left(\mu, B_{\perp}\right)$ phase diagram. The boundaries of the topological phase in the $\left(\mu, B_{\perp}\right)$ plane are given in an analytic form and provide a guideline for the experimental investigation of Majorana bound states in carbon nanotubes.

The paper is structured as follows. Section II contains a description of the microscopic model and Sec. III the description of the four-band effective model and of the nature of the superconducting pairing between different bands. The discussion of the system's symmetries and topological invariants can be found in Sec. IV, while Sec. V holds the analysis of the zero-energy eigenstates, in particular proving them to be true MQPs. In Sec. VI, we study the stability of MQP states against disorder and misalignment of the magnetic field. The last section contains a short summary of our paper and concluding remarks.

Finally, Appendix A contains a description of the valley mixing arising from the presence of a substrate, Appendix B an extended discussion of the system's symmetries, and Appendix $C$ the description of the method by which the boundaries of the topological phases were calculated analytically. In Appendix D, we derive an effective two-band model valid 
at low magnetic fields, and in Appendix E, we shortly discuss the influence of the nearest-neighbor superconducting pairing.

\section{MICROSCOPIC MODEL}

Carbon nanotubes can be regarded as graphene sheets rolled into seamless cylinders. The rolling direction is described by the so-called chiral indices of the CNT, $(n, m)$ [16]. The bulk spectrum of the CNT consists of 1D subbands created by cutting graphene's dispersion by lines of constant angular momentum, determined by the periodic boundary conditions around the circumference. The electronic properties of the nanotube depend strongly on the rolling direction, which decides whether the subbands cross the Dirac points or not. If they do, i.e., when $(n-m) \mid \bmod 3=0$, the nanotubes are metallic and the lowest $1 \mathrm{D}$ conduction bands descend deeply towards the apex of graphene's Dirac cones, reaching Fermi velocities of the order of $10^{6} \mathrm{~m} / \mathrm{s}$. If $(n-m) \mid \bmod 3 \neq 0$, the CNT is semiconducting and the lowest bands lie higher up on the Dirac cones and are much flatter, with Fermi velocities dependent on the chemical potential, but typically not higher than $\sim 10^{3} \mathrm{~m} / \mathrm{s}$. In the following, we shall use for illustration a finite $(12,4) \mathrm{CNT}$, although we find the same topological phases in semiconducting nanotubes of other chiralities, in different parameter regimes.

The microscopic model of the nanotube that we use, with one $p_{z}$ orbital per atomic site, is shown schematically in Fig. 1(a). The model is constructed for one $p_{z}$ orbital per atomic site. The hopping matrix elements, taking into account the hybridization between $\sigma$ and $\pi$ orbitals and the spin-orbit coupling induced by the curvature, are given by the formulas in Refs. [22,23]. In our calculations, we chose $V_{p p}^{\sigma}=6.38 \mathrm{eV}$ and $V_{p p}^{\pi}=-2.66 \mathrm{eV}$ after Ref. [24], and we set the small parameter controlling nanotube's spin-orbit coupling to $\delta_{\mathrm{SO}}=3 \times 10^{-3}$, similar to $2.8 \times 10^{-3}$ measured in Ref. [25].

The tiny spin-orbit coupling of graphene becomes significantly enhanced in carbon nanotubes due to the curvature of their atomic lattice [22,26-28]. It defines a quantization axis for the spin, along the CNT axis, and induces a band splitting

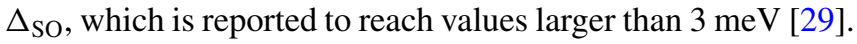
The resulting low-energy band structure for a $(12,4)$ semiconducting nanotube is shown in the small panel of Fig. 1(b) and, zoomed up around the $\Gamma$ point, with the grey lines in the larger panel. The band crossing at $k=0$ is protected by symmetry since the crossing bands belong to different valleys $K$ and $K^{\prime}$, i.e., in this CNT to different angular momenta [30,31], and the magnetic field cannot hybridize them. The presence of a superconducting substrate plays here a double role. On the one hand, it serves as a source of superconducting correlations in the nanotube, acquired by the proximity effect. On the other hand, it breaks the rotational symmetry of the nanotube and is the cause of valley mixing $\Delta_{K K^{\prime}}$. In combination with the perpendicular magnetic field $B_{\perp}$, this allows the bands at the $\Gamma$ point to hybridize. The increased electrostatic potential in the vicinity of the substrate atoms is shown as a darker stripe across the inset in Fig. 1(a). The real space CNT Hamiltonian in the presence of perpendicular magnetic field $B_{\perp}$ is then given by

$$
\begin{aligned}
H_{0}= & \sum_{\langle i, j\rangle, s s^{\prime}} t_{i j, s s^{\prime}} c_{i s}^{\dagger} c_{j s^{\prime}}+\sum_{i, s} V\left(\varphi_{i}\right) c_{i s}^{\dagger} c_{i s} \\
& +\mu_{B} B_{\perp} \sum_{i, s} c_{i s}^{\dagger} c_{i,-s},
\end{aligned}
$$

where $i$ indexes the atomic positions, $s$ is the spin, $t_{i j, s s^{\prime}}$ is the spin-dependent nearest-neighbor hopping [22], $\langle i, j\rangle$ denotes a sum over the nearest-neighbor atoms, and $V\left(\varphi_{i}\right)$ is the potential induced by the substrate at the $i$ th nanotube atom. It depends on the atom's height above the substrate, i.e., on its angular coordinate $\varphi_{i}$. Further details can be found in Appendix A. The resulting band structure is shown in the left large panel of Fig. 1(b), featuring both the helical, spin-momentum locked modes and two energy ranges with odd number of Fermi surfaces. We have also constructed a four-band effective model in the reciprocal space, discussed in the next section, with the band structure shown also in Fig. 1(b). A very good agreement with the spectrum obtained from the full tight-binding calculation is achieved, which is crucial in the studies of topological matter.

When the substrate turns superconducting, it induces Cooper pairing in the nearby normal system. We propose to use the two-dimensional (2D) gate-tunable superconductor $\mathrm{NbSe}_{2}$, where superconductivity can survive up to $30 \mathrm{~T}$ in magnetic fields applied in-plane [32]. Hence in our setup the magnetic field is applied in the direction perpendicular to the nanotube axis but, crucially, parallel to the substrate. We treat the superconducting correlations in the spirit of Ref. [33], admitting both the on-site and nearest-neighbor pairing $\Delta_{0}$ and $\Delta_{1}$. With the superconducting pairing the system is described by

$$
\begin{aligned}
H= & H_{0}-\mu \sum_{i, s} c_{i s}^{\dagger} c_{i s}+\sum_{i, s}\left(\Delta_{0} c_{i s}^{\dagger} c_{i,-s}^{\dagger}+\text { H.c. }\right) \\
& +\sum_{\langle i, j\rangle, s}\left(\Delta_{1} c_{i s}^{\dagger} c_{j,-s}^{\dagger}+\text { H.c. }\right),
\end{aligned}
$$

where energies are measured from the chemical potential $\mu$, controlled, e.g., by a gating layer beneath the substrate. The $\Delta_{1}$ contribution is not necessary for the MQPs to arise and we shall discuss its effects further only in Appendix E, here assuming $\Delta_{0} \in \mathbb{R}$ and $\Delta_{1}=0$. In our calculation, $\Delta_{0}=0.4 \mathrm{meV}$, consistent with the value of the gap reported in proximitized CNT quantum dots [34]. In order to find the spectrum of a superconducting CNT, we express the Hamiltonian (2) in a particle-hole symmetric form by introducing a Nambu spinor, $\Psi=\bigoplus_{i=1}^{N} \Psi_{i}, \quad \Psi_{i}^{\dagger}=\left(c_{i \uparrow}^{\dagger}, c_{i \downarrow}^{\dagger}, c_{i \uparrow}, c_{i \downarrow}\right)$, where $\oplus$ is the direct sum over the $N$ atomic positions [35]. This procedure effectively doubles the number of degrees of freedom of the system. The full Hamiltonian becomes $H=\frac{1}{2} \Psi^{\dagger} H_{\mathrm{BdG}} \Psi$, where the field operators are contained in $\Psi, \Psi^{\dagger}$ and $H_{\mathrm{BdG}}$ is an ordinary matrix, the Bogoliubov-de Gennes Hamiltonian of our system. Its eigenvectors, defining the quasiparticle eigenstates with a set of quantum numbers $n$, have the structure

$$
\chi^{n}=\oplus_{i=1}^{N} \chi_{i}^{n}, \quad\left(\chi_{i}^{n}\right)^{T}=\left(u_{i \uparrow}^{n}, u_{i \downarrow}^{n}, v_{i \uparrow}^{n}, v_{i \downarrow}^{n}\right),
$$

where $n$ is a generic collective index, which may contain, e.g., the valley and, in a system with translational invariance, 


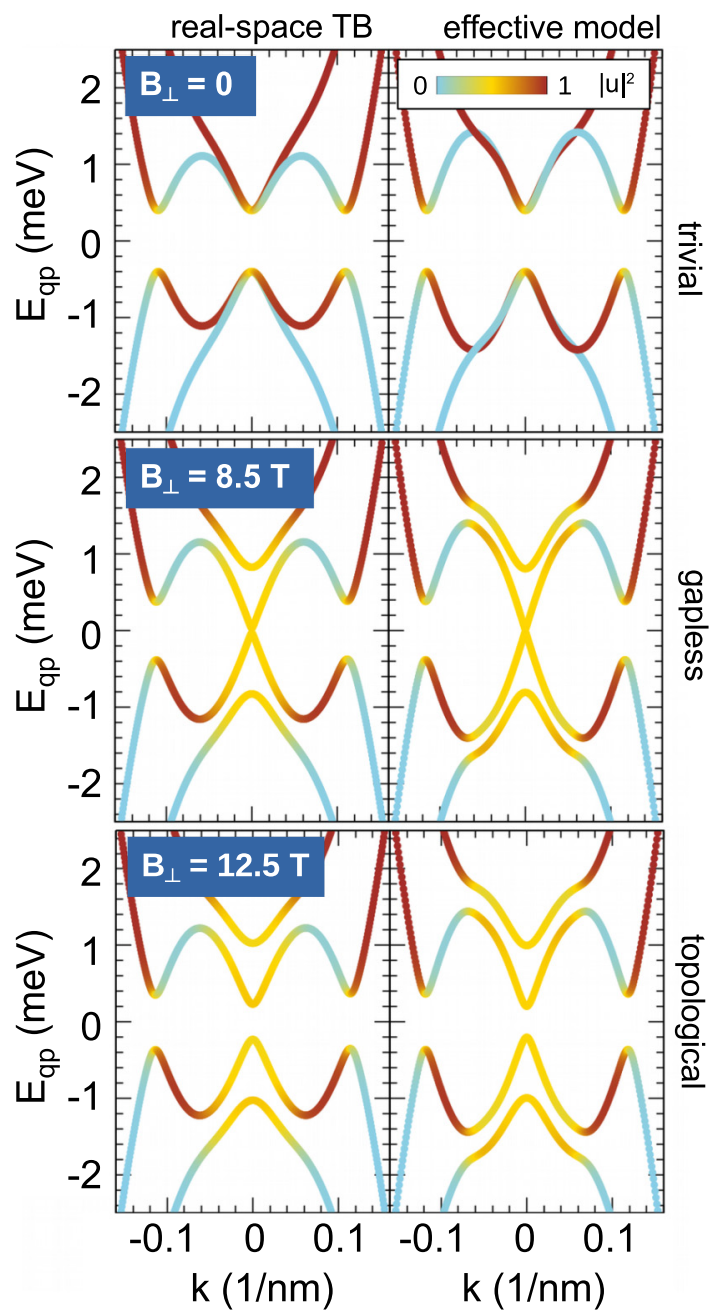

FIG. 2. The Bogoliubov-de Gennes spectra of the superconducting nanotube in three different topological phases, which can be accessed by tuning $B_{\perp}$ for the chemical potential $\mu=334.6 \mathrm{meV}$. The color scale shows the weight of the particle part of the corresponding CNT's eigenstate; gold color indicates equal particle and hole contributions. The superconducting pairing is $\Delta_{0}=0.4 \mathrm{meV}$, $\Delta_{1}=0$.

$k$ quantum numbers. The particle components with spin $s$ on atom $i$ are denoted by $u_{i s}^{n}$ and the corresponding hole components by $v_{i s}^{n}$. The quantum eigenstates of the system have the form $\left|\psi^{n}\right\rangle=\oplus_{i=1}^{N} \Psi_{i}^{\dagger} \cdot \chi_{i}^{n}|0\rangle_{\mathrm{BCS}}$, where $|0\rangle_{\mathrm{BCS}}$ is the $\mathrm{BCS}$ ground state in the CNT. The low-energy bands obtained for our proximitized infinite $(12,4)$ nanotube are shown in Fig. 2, for the three topologically distinct phases encountered by increasing the magnetic field. The color scale shows the overall weight of particle component in the given energy eigenstate, $|u|^{2}=\sum_{i s}\left|u_{i s}\right|^{2}$. The solutions, which have a predominantly particle character, trace the original singleparticle bands, while the predominantly hole-type solutions are mirror-reflected around the chemical potential.

\section{EFFECTIVE FOUR-BAND MODEL}

Approximate models in the reciprocal space can be helpful to get a better physical insight regarding the underlying sym- metries and processes driving the topological phase transitions; furthermore, they enable us to calculate the topological phase diagram analytically. In this section, an effective four-band model is obtained starting from Eq. (2), with parameters fitted against the numerically calculated spectrum. We advance the analysis in Ref. [21] by discussing in detail the discrete symmetries of our model and the associated topological invariants. Furthermore, an analytical expression for the boundaries of the topological phase is provided and the $p$-wave character of the relevant intraband coupling is checked.

The Hamiltonian of a CNT in the reciprocal space is obtained using a zone folding technique. The spectrum of the CNT follows from that of graphene by imposing the periodic boundary conditions on the value of transverse momentum, turning the 2D dispersion of graphene into a series of 1D cuts, which are the CNTs one-dimensional subbands [16]. When the curvature of the CNT's lattice is included, it results in both spin-dependent and spin-independent modifications of graphene's dispersion. They are most significant near the Dirac points of the spectrum. In models treating one $p_{z}$ orbital per atomic site, their effects can be incorporated in the dispersion as shifts in both transverse and longitudinal momentum. The low-energy electronic spectrum of a CNT in the conduction band for given transverse momentum $k_{\perp}$ and longitudinal momentum $k$ is then given by

$$
\begin{aligned}
\varepsilon_{\tau \sigma}\left(k_{\perp}, k\right)= & \hbar v_{F}\left\{\left(k-\tau K_{\|}+\tau \Delta k_{\|}^{c}\right)^{2}\right. \\
& \left.+\left(k_{\perp}-\tau K_{\perp}+\tau \Delta k_{\perp}^{c}+s \Delta k_{\perp}^{\mathrm{SO}}\right)^{2}\right\}^{1 / 2},
\end{aligned}
$$

where $K_{\perp}, K_{\|}$are the transverse and longitudinal component of momentum at the Dirac point $K$. The quantum numbers $\tau$ and $s$ stand for the valley $\left(K: \tau=1, K^{\prime}: \tau=-1\right)$ and the spin component along the CNT axis $s= \pm 1$. All quantities in this dispersion are directly related to the hopping integrals across $\pi\left(V_{p p}^{\pi}\right)$ and $\sigma$ bonds $\left(V_{p p}^{\sigma}\right)$ in graphene, to nanotube geometry, and to carbon's intrinsic spin-orbit coupling [22,23], and their values and signs may vary, depending on which set of tightbinding parameters is used. The numerical values of those momentum shifts in our calculations are $\Delta k_{\perp}^{c}=-22.83 \mu \mathrm{m}^{-1}$, $\Delta k_{\|}^{c}=66.62 \mu \mathrm{m}^{-1}$, and $\Delta k_{\mathrm{SO}}=-2.917 \mu \mathrm{m}^{-1}$. In the case of our $(12,4)$ semiconducting nanotube, $K_{\|}=0$ and the lowest energy subbands shown in Fig. 1(b) have $k_{\perp}-\tau K_{\perp}=\tau / 3 R$. In the following, we shorten the notation by setting $k_{\perp}=K_{\perp}+$ $1 / 3 R$ and omitting it from the argument of $\varepsilon_{\tau s}\left(k_{\perp}, k\right)$. The spin-orbit splitting $\Delta_{\mathrm{SO}}$ is defined as $\Delta_{\mathrm{SO}}=\varepsilon_{K \uparrow}(0)-\varepsilon_{K \downarrow}(0)$. Note that the single-particle energies satisfy the time-reversal conjugation, $\varepsilon_{\tau s}(k)=\varepsilon_{-\tau-s}(-k)$.

With added valley-mixing induced by the superconducting substrate and in an external perpendicular magnetic field, the CNT is described by the following effective Hamiltonian:

$$
H=H_{\mathrm{CNT}}+H_{\Delta_{K K^{\prime}}}+H_{\mathrm{Z}} \text {. }
$$

The effective Hamiltonian in second quantization for the CNT including a reference chemical potential $\mu$ is given by

$$
H_{\mathrm{CNT}}-\mu N=\sum_{k, \tau, s} \xi_{\tau s}(k) c_{k \tau s}^{\dagger} c_{k \tau s},
$$

where $\xi_{\tau s}(k)$ is the single-particle energy measured with respect to the chemical potential, $\xi_{\tau s}(k)=\varepsilon_{\tau s}(k)-\mu$. We model the $k$ dependence of the valley mixing 
potential (see Appendix A for details) by modifying the longitudinal curvature shift and fitting an appropriate constant $\Delta_{K K^{\prime}}$ to the band structure obtained from the real space calculation. In our case, $\varepsilon_{\tau s}(k)=$ $\hbar v_{F} \sqrt{\left(k+0.8 \tau \Delta k_{\|}^{c}\right)^{2}+\left(1 /(3 R)+\Delta k_{\perp}^{c}+\tau s \Delta k_{\perp}^{\mathrm{SO}}\right)^{2}}$. The valley-mixing term $H_{\Delta_{K K^{\prime}}}$ couples two electron states at opposite valleys but with the same spin $s$ and becomes

$$
H_{\Delta_{K K^{\prime}}}=\sum_{k, s} \Delta_{K K^{\prime}} c_{k K s}^{\dagger} c_{k K^{\prime} s}+\Delta_{K K^{\prime}}^{\star} c_{k K^{\prime} s}^{\dagger} c_{k K s},
$$

with $\Delta_{K K^{\prime}} \in \mathbb{C}$. In our calculations $\Delta_{K K^{\prime}}$ is real and equal to $2.5 \mathrm{meV}$. The Zeeman energy $H_{Z}$ due to the perpendicular magnetic field $B_{\perp}$ induces a coupling of electrons with opposite spins and in the same valley

$$
H_{Z}=\mu_{B} B_{\perp} \sum_{k, \tau} c_{k \tau \uparrow}^{\dagger} c_{k \tau \downarrow}+c_{k \tau \downarrow}^{\dagger} c_{k \tau \uparrow},
$$

i.e., we assume $B_{\perp}$ to be applied in the $x$ direction, while the $z$ direction runs along the CNT axis. The eigenstates of the resulting Hamiltonian are then in general linear combinations of all $\tau, s$ eigenstates of the original $H_{\mathrm{CNT}}$. We denote them by (1), (2), (3), (4), shown in Fig. 1(b).

The superconducting correlations induced by proximity are treated in a mean-field approximation according to Eq. (2). We only consider the case of an on-site pairing potential which is described by the superconducting gap $\Delta_{0}$. Since $\Delta_{0}$ is isotropic in momentum space, our mean-field pairing Hamiltonian has an $s$-wave gap symmetry. The mean-field Hamiltonian reads

$$
H_{\mathrm{SC}}=\sum_{k} \Delta_{0}\left(c_{k K \uparrow}^{\dagger} c_{-k K^{\prime} \downarrow}^{\dagger}+c_{k K^{\prime} \uparrow}^{\dagger} c_{-k K \downarrow}^{\dagger}+\text { H.c. }\right),
$$

where we are coupling the corresponding Kramers partners. Introducing the Nambu spinor defined as

$$
\Psi^{\dagger}=\left(c_{k K \uparrow}^{\dagger}, c_{k K \downarrow}^{\dagger}, c_{k K^{\prime} \uparrow}^{\dagger}, c_{k K^{\prime} \downarrow}^{\dagger}, c_{-k K^{\prime} \downarrow}, c_{-k K^{\prime} \uparrow}, c_{-k K \downarrow}, c_{-k K \uparrow}\right),
$$

we obtain the Bogoliubov-de Gennes (BdG) Hamiltonian

$$
\mathcal{H}_{\mathrm{BdG}}(k)=\left(\begin{array}{cc}
H(k) & \Delta \\
\Delta & -H(k)
\end{array}\right),
$$

with

$$
H(k)=\left(\begin{array}{cccc}
\xi_{K \uparrow}(k) & \mu_{B} B_{\perp} & \Delta_{K K^{\prime}} & 0 \\
\mu_{B} B_{\perp} & \xi_{K \downarrow}(k) & 0 & \Delta_{K K^{\prime}} \\
\Delta_{K K^{\prime}} & 0 & \xi_{K^{\prime} \uparrow}(k) & \mu_{B} B_{\perp} \\
0 & \Delta_{K K^{\prime}} & \mu_{B} B & \xi_{K^{\prime} \downarrow}(k)
\end{array}\right)
$$

and

$$
\Delta=\left(\begin{array}{cccc}
-\Delta_{0} & & & \\
& \Delta_{0} & & \\
& & -\Delta_{0} & \\
& & & \Delta_{0}
\end{array}\right) .
$$

The single-particle energies are defined with respect to the chemical potential $\mu$, as in (6). When expressed in the eigenbasis of the single-particle Hamiltonian (5), the superconducting pairing couples all four bands, though not with equal strength. The most important are the intraband pairing and interband pairing within the same pair. Reflecting the same spin direction $s_{x}$ between $k$ and $-k$ states in the same band, the intraband pairing is odd in $k$, thus we call it $\Delta_{p}$. The interband pairing is even in $k$ and we call it $\Delta_{s}$. Analytical expressions for $\Delta_{s}$ and $\Delta_{p}$, derived with the assumption that the two band pairs are decoupled (valid in low fields), are given in Appendix D. The pairings with the members of the other band pair are weaker, and we call them $\Delta_{p}^{\prime}$ and $\Delta_{s}^{\prime}$. The different pairings coupling a band (1) state with positive $k$ to the states with negative $k$ are illustrated in Fig. 3(a), and their $k$ dependence at $B_{\perp}=10 \mathrm{~T}$ is plotted in Fig. 3(b).

The dependence of those different pairings on $k$ and $B_{\perp}$ is plotted in Fig. 3(c). Initially, with increasing field strength, the spins become polarized in the $x$ direction, thus the terms $\Delta_{p}, \Delta_{s}^{\prime}$ pairing the same $s_{x}$ states become on average weaker, while $\Delta_{s}, \Delta_{p}^{\prime}$ pairing opposite $s_{x}$ states gain in strength. Beyond the field strength of $\sim 20 \mathrm{~T}$, the amplitude of the Zeeman term $\mu_{B} B_{\perp}$ becomes comparable to that of the spin-orbit splitting and the pairing $\Delta_{s}^{\prime}$ mixes the two band pairs. This effect will be visible in the topological phase diagram discussed in the next section.

The region that holds greatest interest for the experimental realizations is that of lower magnetic fields, in the neighborhood of $B_{c}$, i.e., the lowest field for which the energy gap closes at the $\Gamma$ point (given by $\mu_{B} B_{c}=2 \Delta_{0} \Delta_{K K^{\prime}} / \sqrt{\Delta_{\text {SO }}^{2}+4 \Delta_{K K^{\prime}}^{2}}$ ). In this regime, the two band pairs can be considered independent, and we show in the next section that near the critical field they give the largest contribution to the topological phase. The construction of this further simplified model is described in Appendix D, allowing us to find analytically the energy spectrum.

\section{SYMMETRIES AND TOPOLOGICAL INVARIANTS}

The Hamiltonian $H_{\mathrm{BdG}}$, like all Bogoliubov-de Gennes Hamiltonians, is by construction invariant under a particlehole operation. That is, we can define an antiunitary operator $\mathcal{P}$, such that $\mathcal{P} H_{\mathrm{BdG}} \mathcal{P}^{-1}=-H_{\mathrm{BdG}}$. The action of $\mathcal{P}$ on the original electron operators and on doubled Hilbert space states is

$$
\mathcal{P} c_{i s}=c_{i s}^{\dagger}, \quad \mathcal{P} \chi_{i}=\left(v_{i \uparrow}^{*}, v_{i \downarrow}^{*}, u_{i \uparrow}^{*}, u_{i \downarrow}^{*}\right)^{T} .
$$

The particle-hole operation maps the positive energy solutions onto their Nambu partners with negative energy. If the particlehole symmetric Hamiltonian of a finite system has zero-energy modes, they can be cast in the form of eigenstates of $\mathcal{P}$,

$$
\mathcal{P} \psi=\psi \text {. }
$$

Inspecting the first relation of (12) shows that (13) is only an equivalent definition of the Majorana property, usually stated as $\gamma_{\sigma}(\boldsymbol{r})=\gamma_{\sigma}^{\dagger}(\boldsymbol{r})$, where $\gamma^{\dagger}$ is the operator creating a particle with spin $\sigma$ at position $\boldsymbol{r}$.

The presence or absence of Majorana solutions can be predicted from a topological phase diagram, where different phases correspond to different values of a topological invariant. In a system with translational symmetry, such as the bulk of the CNT, the basic quantity determining the topological invariant in 1D is $\gamma^{-}$, the sum of the Berry phases carried by all occupied (negative energy) bands, integrated over the Brillouin zone. Since $\gamma^{-}$is gauge-dependent and defined only up to an integer, another invariant is commonly used, $W=\exp \left(i 2 \pi \gamma^{-}\right)$, which is gauge-independent. The particlehole symmetry in a system with translational invariance is 

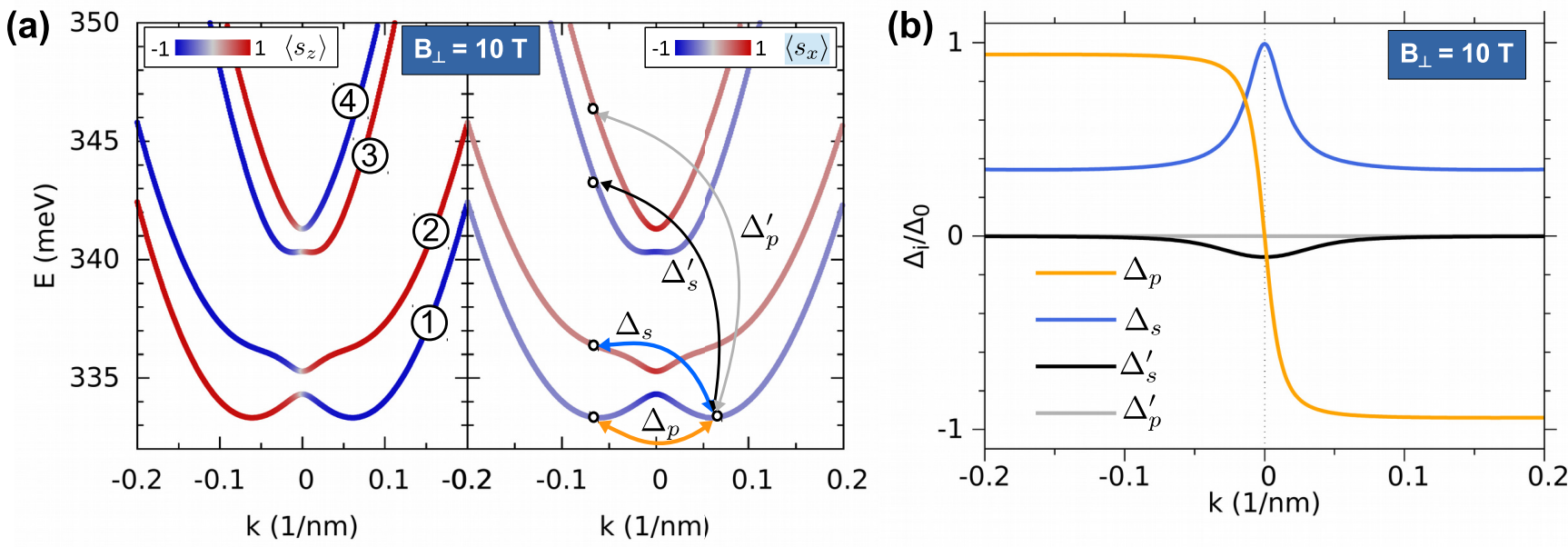

(c)
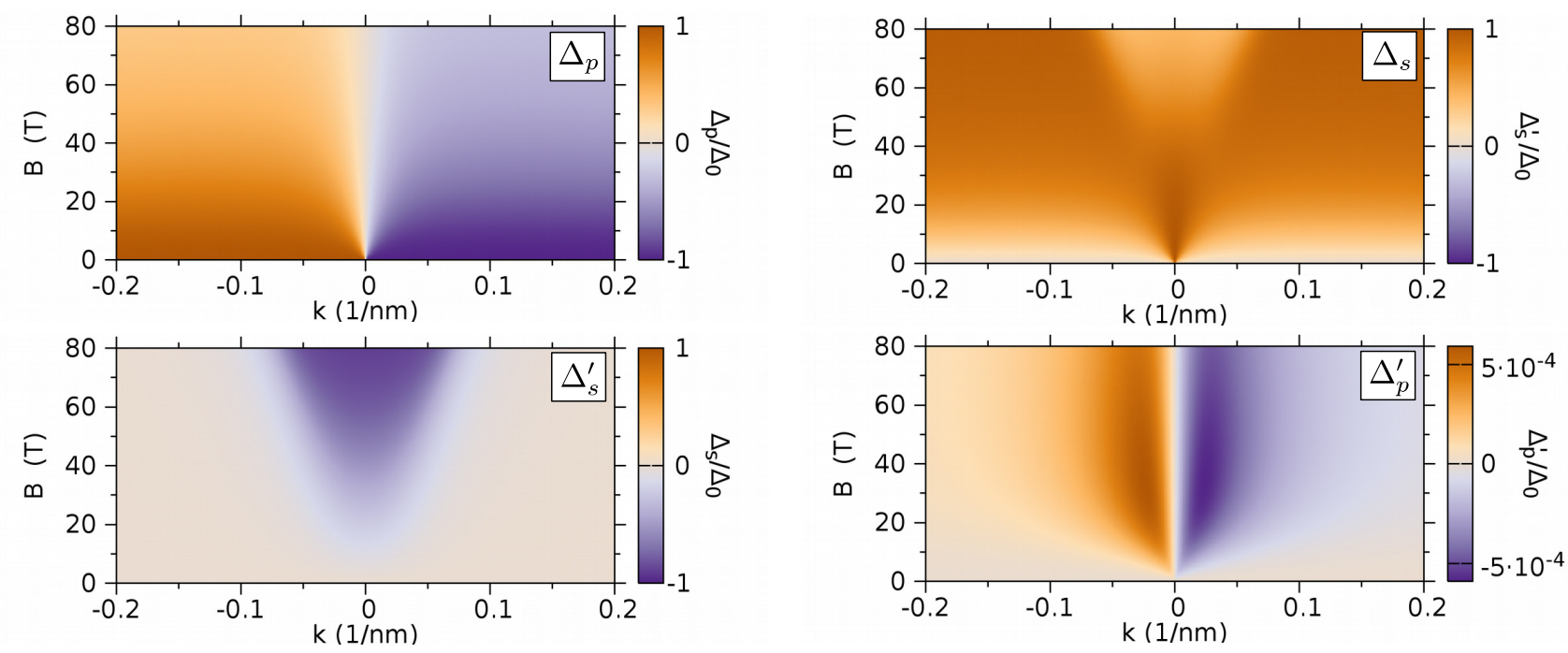

FIG. 3. (a) The lowest four bands of a $(12,4)$ semiconducting CNT with valley mixing and in $B_{\perp}=10 \mathrm{~T}$, obtained with the effective four-band model. The color scale shows the expectation value of the $s_{z}$ (left panel) or $s_{x}$ (right panel) component of an eigenstate's spin. The pairings between a positive $k$ state in band (1) and the four states with opposite $k$ are indicated in the right panel. (b) Pairing strength as a function of $k$ for $B_{\perp}=10 \mathrm{~T}$, in $\Delta_{0}$ units. (c) The four pairing terms as functions of $k$ and $B_{\perp}$, in $\Delta_{0}$ units. Note the increase in $\Delta_{s}^{\prime}$, which couples the upper and lower band pairs, beyond $\sim 20 \mathrm{~T}$.

expressed as $\mathcal{P} H_{\mathrm{BdG}}(k) \mathcal{P}^{-1}=-H_{\mathrm{BdG}}(-k)$ [36,37], i.e., the positive energy solutions at momentum $k$ are related to negative energy solutions at momentum $-k$, as sketched in Fig. 4(a). This constrains the values which $W$ can take to \pm 1 , i.e., $W$ is of a $\mathbb{Z}_{2}$ type, associated with the Altland-Zirnbauer D class systems [36,37]. $W=+1$ corresponds to the trivial topological phase, while $W=-1$ implies the presence of MQPs at the system boundaries. The phase diagram calculated for our model nanotube, using the standard Pfaffian technique $[21,36]$ and the effective model for the bulk bands, is shown in Fig. 4(b). The borders between different phases in the diagram correspond to $\left(B_{\perp}, \mu\right)$ such that the gap is closed at $k=0$. From our effective four-band model, we find that this occurs at

$$
\begin{aligned}
\tilde{\mu}^{2}= & \Delta_{\mathrm{SO}}^{2} / 4+\left(\Delta_{K K^{\prime}}^{2}+\left(\mu_{B} B_{\perp}\right)^{2}-\Delta_{0}^{2}\right) \\
& \pm \sqrt{4 \Delta_{K K^{\prime}}^{2}\left(\left(\mu_{B} B_{\perp}\right)^{2}-\Delta_{0}^{2}\right)-\Delta_{0}^{2} \Delta_{\mathrm{SO}}^{2}},
\end{aligned}
$$

where $\tilde{\mu}$ is the chemical potential measured from the center of either the (1), (2) or (3), (4) pair in Fig. 1(b). The critical magnetic field is given by $\mu_{b} B_{c}=\Delta_{0} \sqrt{4 \Delta_{K K^{\prime}}^{2}+\Delta_{\mathrm{SO}}^{2}} /\left(2 \Delta_{K K^{\prime}}\right)$. If we assume that the band pair (1), (2) is independent of (3), (4) (i.e., $\Delta_{s}^{\prime}$ can be neglected), we can expand (14) around $B_{c}$, obtaining a simpler formula $\tilde{\mu}^{2}=\Delta_{0}^{2}\left(\left(B_{\perp} / B_{c}\right)^{2}-1\right)$. The red lines in Fig. 4(b) follow (14), the dashed lines mark the borders of the nontrivial phase obtained with the simpler approximated formula. The coupling between the band pairs changes visibly the phase diagram-when the Zeeman energy reaches the magnitude of the original spin-orbit splitting, it destroys the topological phase. The same phenomenon occurs in multiband semiconducting nanowires, where the mixing between various transverse modes caused by the Rashba spin-orbit coupling strongly reduces the nontrivial topological regions in the phase diagram $[11,38,39]$.

As can be seen in Fig. 2, the Hamiltonian $H_{\mathrm{BdG}}$ is highly symmetric. In particular, a unitary operation $C$ can be defined, 
(a)

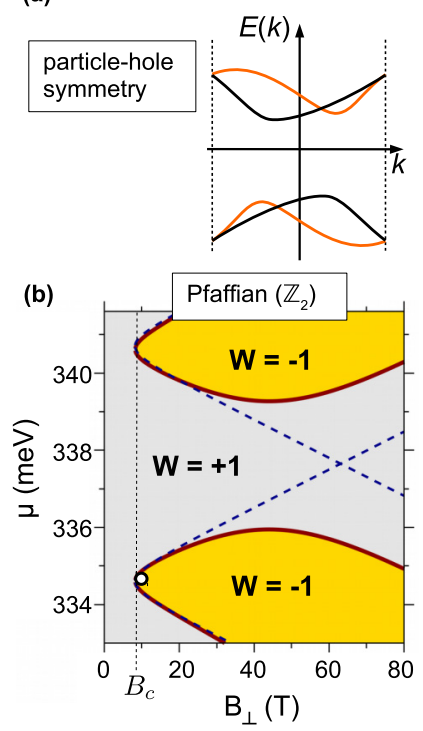

(c)
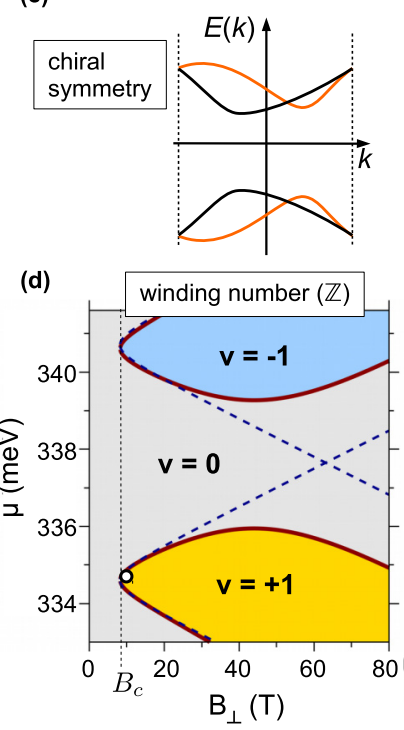

FIG. 4. Symmetries and topological invariants. (a) Sketch of a spectrum with particle-hole symmetry. Bands of the same color are related by the symmetry. (b) The phase diagram calculated using the effective model and the Pfaffian formulation of the topological invariant, typical for particle-hole symmetric systems. The topologically nontrivial regions are shown in yellow, the red line at the border between the phases is the contour of $E=0$ at the $\Gamma$ point. The dot in the lower $W=-1$ area marks the $\mu$ and $B_{\perp}$ used in Fig. 6. The dashed lines trace the borders of nontrivial phase calculated from a model which contains only one single-particle band pair, either (1) and (2) (lower region) or (3) and (4) (higher region) from Fig. 1(b). (c) Sketch of a spectrum with chiral symmetry. The Bogoliubov-de Gennes spectrum in Fig. 2 has both particle-hole and chiral symmetry. (d) The phase diagram calculated using the winding number invariant, defined for chiral symmetric systems. The values $v= \pm 1$ in the lower and upper nontrivial area indicate that these regions correspond to different topological phases, with one zero-energy mode in each.

such that $C H_{\mathrm{BdG}}(k) C^{-1}=-H_{\mathrm{BdG}}(k)$. The operation $C$ is a so-called chiral symmetry, connecting positive and negative energy solutions at the same momentum $k$, as sketched in Fig. 4(c). The MQPs in our system are also eigenstates of $C$. In systems with this symmetry, the topological invariant $\gamma^{-}$has a clear interpretation as a winding number, $\gamma^{-}=v / 2$ [40]. The winding number is an integer, i.e., it belongs to $\mathbb{Z}$. That apparent contradiction with $W \in \mathbb{Z}_{2}$ is solved when we recall that $W$ was constructed with an extra exponentiation step, which obliterates the difference between the phases with $v= \pm 1$. The phase diagram calculated using the winding number is shown in Fig. 4(d), with exactly the same phase boundaries, but showing clearly that the lower nontrivial region and the upper nontrivial region in fact correspond to different nontrivial phases. Further discussion of the symmetries and both invariants can be found in Appendix B.

With both the particle-hole $\mathcal{P}$ and the chiral $C$ symmetries, the Hamiltonian is also invariant under a product of both, i.e., $\tilde{\mathcal{T}}=C \mathcal{P}^{-1}$. This symmetry is antiunitary and commutes with the Hamiltonian, $\tilde{\mathcal{T}} H_{\mathrm{BdG}}(k) \tilde{\mathcal{T}}^{-1}=H_{\mathrm{BdG}}(-k)$, similar to the time-reversal. Contrary to the true time-reversal operation which in systems with half-integer spin squares to -1 , here
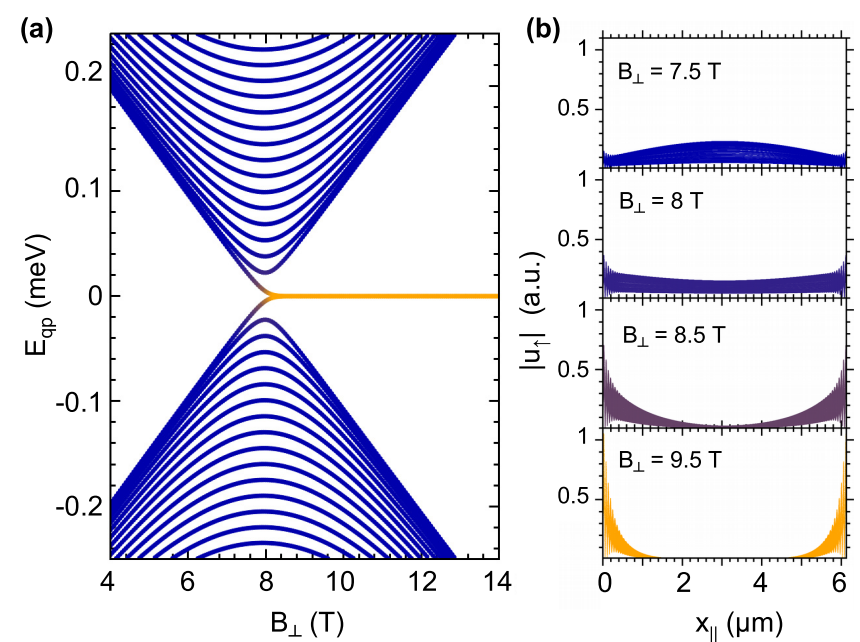

FIG. 5. Topological phase transition. (a) The quasiparticle spectrum of a finite $(12,4)$ nanotube with 4000 unit cells $(L=6.03 \mu \mathrm{m})$, at the chemical potential $\mu=334.6 \mathrm{meV}$ for varying magnetic field. The topological phase transition occurs at $B_{c}=8.5 \mathrm{~T}$, beyond which the lowest energy eigenstate becomes a zero-energy mode. (b) The wave function of the lowest energy mode undergoes a gradual localization with increasing magnetic field. Here only the amplitude $\left|u_{\uparrow}\left(x_{\|}\right)\right|$of the spin up particle component, projected onto the direction along the CNT's axis, is shown. The shape of the remaining components is indistinguishable from that of $\left|u_{\uparrow}\left(x_{\|}\right)\right|$at this scale, which comprises the data points from $N=8.32 \times 10^{5}$ atoms. The units are arbitrary and the same for all wave function plots in this figure.

$\tilde{\mathcal{T}}^{2}=+1$, placing our nanotube not in the $\mathrm{D}$, but in the BDI class with the winding number as an integer topological invariant. Physically, $\tilde{\mathcal{T}}$ represents the rotation of the system by $\pi$ around the axis perpendicular to both the CNT and the substrate.

\section{EMERGENCE OF MQPS IN FINITE NANOTUBES}

Changing the chemical potential or the strength of the magnetic field can drive the proximitized nanotube across a topological phase transition, into a regime in which it becomes a topological superconductor. An example of the changes in the Bogoliubov-de Gennes spectrum during such a transition is shown in Fig. 5(a), for a 6- $\mu$ m-long $(12,4)$ CNT at a fixed chemical potential $\mu=334.6 \mathrm{meV}$ and varying magnetic field $B_{\perp}$. The energy of the lowest quasiparticle states is further lowered with increasing $B_{\perp}$, until they become a doubly degenerate zero-energy mode. The degeneracy is artificial, caused by the doubling of degrees of freedom introduced with the Nambu spinor, and the nanotube de facto hosts only one eigenstate at zero energy. The change in the shape of the quasiparticle wave function associated with the lowest energy eigenstate is illustrated in Fig. 5(b), showing clearly its increasing localization at the ends of the proximitized CNT. In the figure only the amplitude $\left|u_{\uparrow}(\boldsymbol{r})\right|$ of the particle component with spin up is shown, the remaining components $u_{\downarrow}(\boldsymbol{r}), v_{\uparrow}(\boldsymbol{r}), v_{\downarrow}(\boldsymbol{r})$ have profiles which are indistinguishable from $\left|u_{\uparrow}(\boldsymbol{r})\right|$ at this scale. Having a direct access to the particle 
(a)
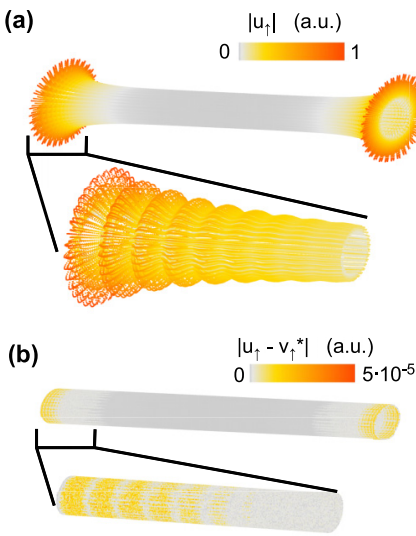

FIG. 6. Majorana bound states. (a) The full spatial profile of the spin up and spin down particle components, $\left|u_{\uparrow}(\boldsymbol{r})\right|$ and $\left|u_{\downarrow}(\boldsymbol{r})\right|$. The amplitude of the electronic wave function is shown through both the distance from the nanotube's surface (light grey) and through the color scale. The wavelength of the oscillations is given by the value of $k_{F}$ at the chosen chemical potential. (b) Spatially resolved amplitude of the difference between the particle and conjugated hole components for the same spin, $\left|u_{\uparrow}(\boldsymbol{r})-v_{\uparrow}^{*}(\boldsymbol{r})\right|$ and $\left|u_{\downarrow}(\boldsymbol{r})-v_{\downarrow}^{*}(\boldsymbol{r})\right|$. The distance from the CNT's surface is scaled in the same way as in (a), and the color scale is greatly enhanced. Only faint differences are visible, of the order of $10^{-5}$, which shows the Majorana nature of the zero-energy mode.

and hole components of the zero-energy mode, we can prove that it indeed has Majorana nature according to (13).

The spatially resolved wave function of the zero-energy mode at $B_{\perp}=9.5 \mathrm{~T}$ is shown in Fig. 6(a). The amplitude of spin-up and -down particle components, $\left|u_{\uparrow}(\boldsymbol{r})\right|$ and $\left|u_{\downarrow}(\boldsymbol{r})\right|$, is shown both as the distance from the CNT's surface (grey) at each atomic position and via the color scale. The wavelength of the oscillations is set by the value of $k_{F}$ at the chosen chemical potential. The decay length is field-dependent, and at $B_{\perp}=$ 9.5 , it is $\sim 0.4 \mu \mathrm{m}$. The Majorana nature of the zero-energy mode becomes evident in the Fig. 6(b), where the differences between particle and (complex conjugated) hole component of the wave function for each spin, $\left|u_{\uparrow}(\boldsymbol{r})-v_{\uparrow}^{*}(\boldsymbol{r})\right|$ and $\mid u_{\downarrow}(\boldsymbol{r})-$ $v_{\downarrow}^{*}(\boldsymbol{r}) \mid$ are shown. They are identical up to the order of $10^{-5}$ of the maximum amplitude, which constitutes a numerical proof that the zero-energy mode fulfills the Majorana condition (13).

Both the Pfaffian and the winding number invariants predict correctly whether the system is in a trivial or nontrivial topological phase, but the winding number also distinguishes between different nontrivial phases. This could be seen from Fig. 4(d), where the upper and lower nontrivial regions are characterized by different values of the winding number. In consequence, if the chemical potential of the CNT is tuned in such a way that a part of the tube resides in the phase with $v=-1$ and another in the $v=+1$ phase, two MQP modes arise, localized at the ends of the CNT and back-to-back at the boundary between the two phases. This situation is shown in Figs. 7(a) and 7(b), where the left half of the CNT is at $\mu_{L}=334.6 \mathrm{meV}$, the right half at $\mu_{R}=340.7 \mathrm{meV}$, the crossover region where the potential varies smoothly from $\mu_{L}$ to $\mu_{R}$ has the length of $\sim 20 \AA$ and the magnetic field is $B_{\perp}=14 \mathrm{~T}$.

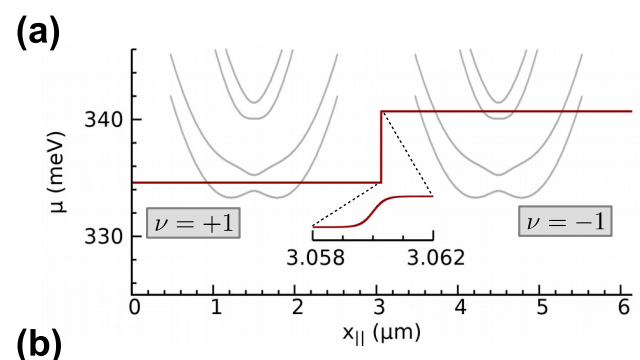

(b)
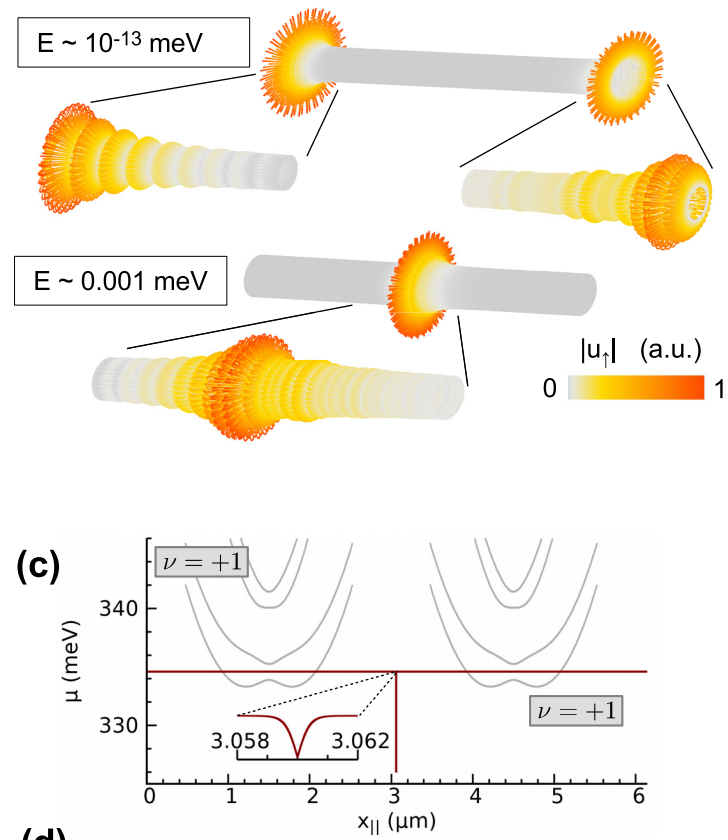

(d)

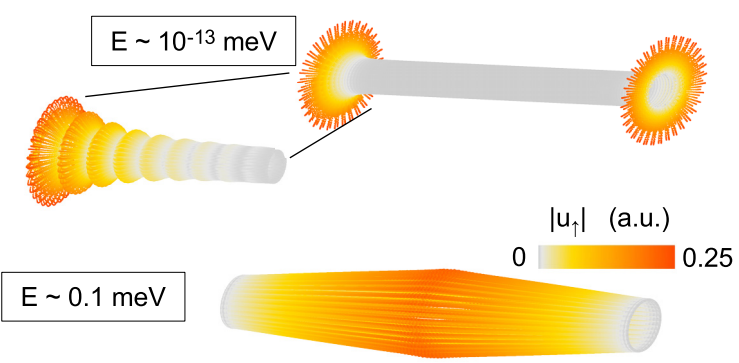

FIG. 7. (a) The profile of the chemical potential where the left and right half of the CNT are in different nontrivial topological phases. The magnetic field is $B_{\perp}=14 \mathrm{~T}$ and the length of the crossover region is $\sim 20 \AA$. The grey lines show for reference the energy bands. (b) The amplitude $\left|u_{\uparrow}(\boldsymbol{r})\right|$ of spin up component of the two lowest energy eigenstates. Remaining components have almost identical profiles. The state localized at the ends is a true MQP. Note different wave function profile at the left and right end, which are in different phases. The eigenstate in the center is composed of the partners of the left and right parts of the Majorana mode, which overlap and slightly hybridize, pushing the state's energy to roughly $1 \%$ of the bulk gap. (c) The chemical potential profile for a CNT whose two halves are in the same phase, but separated by a narrow region of the trivial phase, also with the length of $\sim 20 \AA$. (d) The amplitude $\left|u_{\uparrow}(\boldsymbol{r})\right|$ of spin-up component of the two lowest energy eigenstates. Remaining components have almost identical profiles. The lower energy eigenstate is a Majorana mode, the next one belongs already to the bulk, extending over the whole CNT. 
The lowest energy mode, localized at the CNT ends, is a true Majorana mode with the energy equal zero within the machine precision. Since it is composed of two parts from different topological phases, its wave function has different profile at the left and at the right end. Its left part is characterized by a single characteristic oscillation period, corresponding to the $k_{F}$ of band (1), which is the single one contributing to the zero-energy mode in the lower nontrivial region. The right part of the Majorana mode shows clear beating behavior, due to the interference of contributions from (1), (2), and (3) bands. The other low-energy state is composed of the partners of the left and right part, located at the phase boundary. There the two modes overlap and slightly hybridize, moving the energy of the resulting state to $\sim 1 \%$ of the band gap, and skewing them from the true Majorana nature. The overlap between the two modes has however much more dramatic consequences if the two halves of the CNT are in the same phase, with an equally narrow region of trivial phase in the center, as shown in Figs. 7(c) and 7(d). There the end state remains a Majorana state, but the center state hybridizes fully and moves into the bulk.

\section{MQP STABILITY AND EXPERIMENTAL FEASIBILITY}

\section{A. Disorder}

The stability of the MQPs against perturbations is crucial for their experimental realization. The techniques for growing carbon nanotubes are now so advanced that their atomic lattices are nearly perfect $[41,42]$. Nevertheless, some atoms may be adsorbed on the nanotube during the device production. We simulate their effect through a random on-site electrostatic potential, with varying impurity concentration $n_{0}=N_{\text {imp }} / N$, and potential strength chosen randomly from a range $\left[-W_{0}, W_{0}\right]$. The evolution of the quasiparticle spectra with magnetic field for two impurity concentrations and varying disorder strength is shown in Fig. 8. The nanotube and other parameters $\left(\Delta_{0}, V\left(\varphi_{i}\right)\right)$ remain unchanged.

At realistically low concentrations increasing $W_{0}$ delays the onset of the zero-energy mode, as can be seen from Figs. 8(a) and 8(c), and perturbs the bulk bands [cf. Fig. 8(c) at $W_{0}=0.6$ and $\left.0.9 \mathrm{eV}\right]$. It also decreases the gap between the zero-energy mode and the bulk states [cf. Fig. 8(b)], but the Majorana mode is clearly present and protected, albeit it forms at higher $B_{\perp}$ than in the clean system.

Increasing the impurity concentration beyond the realistic values, to $n_{0}=1 \%$, as illustrated in Figs. 8(d)-8(f), is more effective at destroying the nontrivial topological phase than the increase in the disorder strength. For intermediate and large disorder strength $W_{0} \geqslant 0.5 \mathrm{eV}$ the formation of the zero mode occurs at much higher $B_{\perp}$, as can be seen from Figs. 8(d) and $8(\mathrm{f})$. Even when the zero-energy mode forms, it is mixed with the bulk bands [cf. Figs. 8(e) and 8(f)], resulting in several ordinary localized states.

\section{B. Magnetic field misalignment}

Another factor which has to be taken into account is the precision of alignment of the magnetic field. The presence of a field component parallel to the nanotube axis gives rise to the Aharonov-Bohm effect. In nanotubes this causes a (a)

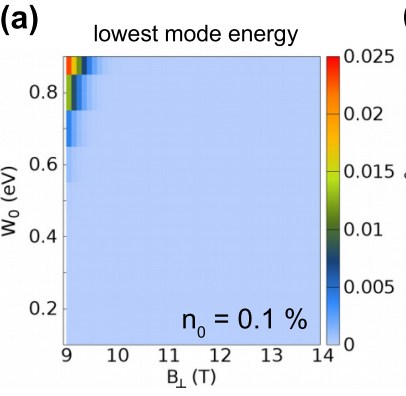

(b)

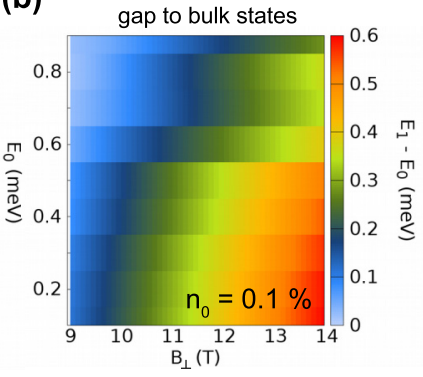

(c)
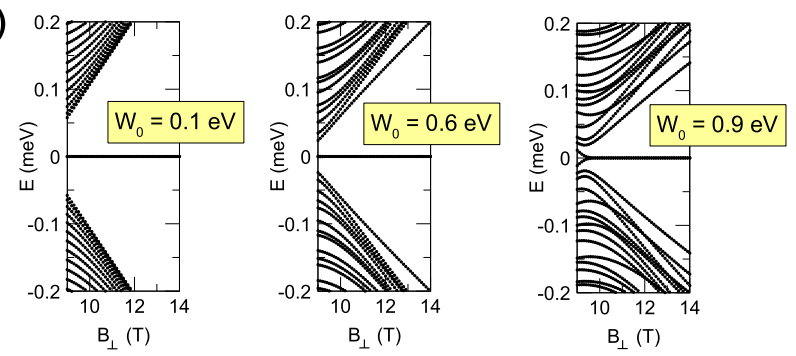

(d)

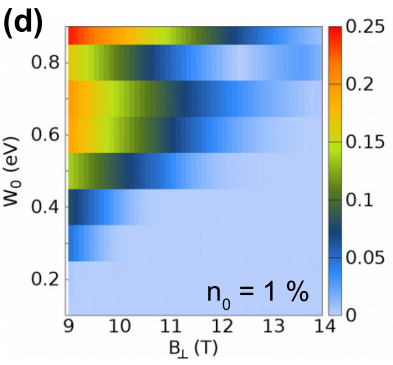

(e)

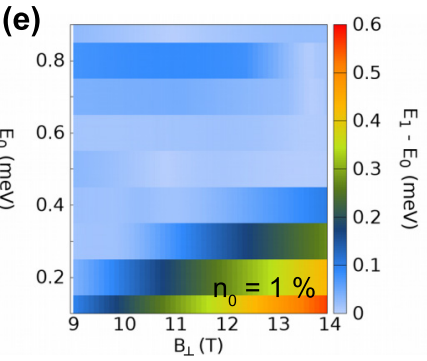

(f)
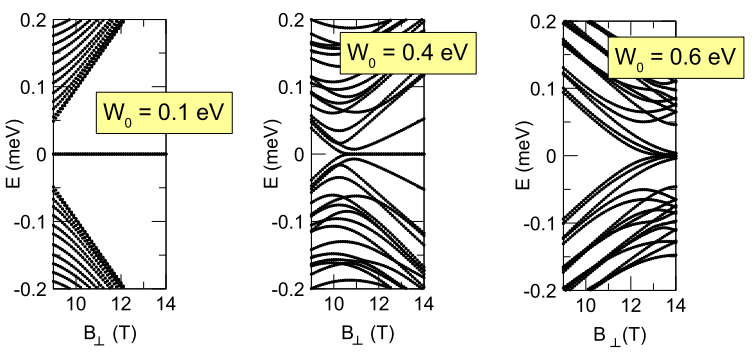

FIG. 8. (a) The energy of the lowest positive mode $E_{0}$ of the $(12,4)$ nanotube with $L \simeq 6 \mu \mathrm{m}$ discussed in the main text. The chemical potential is fixed at $\mu=334.6 \mathrm{meV}$, both the magnetic field $B_{\perp}$ and the maximum disorder strength $W_{0}$ vary. The latter increases in steps of $0.1 \mathrm{eV}$. In this magnetic field range, the clean system is in the nontrivial topological phase. The concentration of impurities in (a)(c) is $0.1 \%$, which corresponds to 832 impurities. In all plots of this figure,each value of $W_{0}$ corresponds to one realization of disorder. (b) The gap between the lowest energy mode $E_{0}$ and the next, $E_{1}$. For $E_{0} \approx 0$, a large value of $E_{1}-E_{0}$ means wide gap between the MQP and the bulk states, indicating a stable MQP mode. (c) Three examples of the quasiparticle spectra near $E=0$. (d) Similar to (a), with a tenfold increase in the impurity concentration, i.e., 8320 impurities in the CNT. The concentration is the same in (d)-(f). (e) Similar to (b). (f) Similar to (c).

different orbital response in the two valleys, resulting in a removal of the valley degeneracy [43] and breaking of the chiral symmetry. When the parallel component of the magnetic field reaches a threshold value, the electrons on opposite sides of the $\Gamma$ point no longer have matching momenta and the 


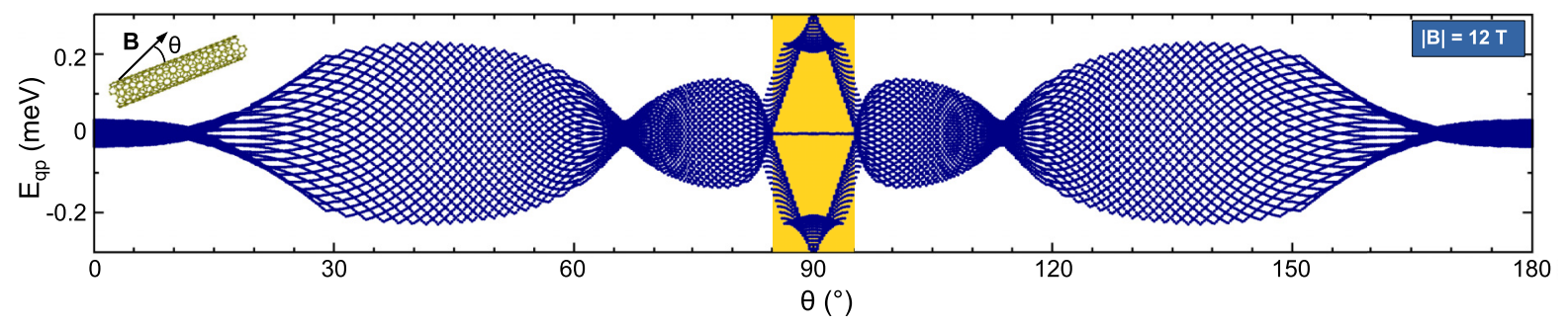

FIG. 9. Stability of MQPs with respect to magnetic field alignment. The thirty two lowest quasiparticle energies as a function of varying angle of the magnetic field, with its amplitude fixed at $B=12 \mathrm{~T}$. The bulk Hamiltonian is gapped only within the area marked in yellow, $85^{\circ} \leqslant \theta \leqslant 95^{\circ}$. In a finite system a zero-energy mode appears throughout this range of $\theta$, with maximum distance to the other eigenstates at $\theta=90^{\circ}$.

superconducting correlations become ineffective, yielding a gapless spectrum. The lowest thirty two eigenvalues of the Bogoliubov-de Gennes spectrum in magnetic field of $12 \mathrm{~T}$ amplitude and varying angle $\theta$ with respect to the nanotube axis are plotted in Fig. 9. At this chosen field amplitude, the finite system supports a Majorana mode within a range of $\pm 5^{\circ}$ deviation of the field from the perpendicular. Increasing the field amplitude widens the maximum gap at $90^{\circ}$, but the higher value of the parallel component decreases the $\theta$ range in which the spectrum is gapped. Maximizing the stability of the MQP in the experiment will then necessarily involve a trade-off between protection against angle fluctuations and protection against scattering into the bulk.

The two major experimental challenges in achieving the formation of MQPs in this setup are the necessity of controlling the chemical potential of the CNT and of applying a large magnetic field without destroying superconducting correlations. Both may be accomplished with the use of $2 \mathrm{D}$ transition metal dichalcogenide (TMDC) superconductors, such as $\mathrm{NbSe}_{2}$, with its larger superconducting gap of $1.26 \mathrm{meV}$ [44]. The superconducting pairing was demonstrated to survive in fields up to $30 \mathrm{~T}$ [32], and the thinness of the 2D layer allows the superconductor itself to be gated, together with the CNT in its proximity.

\section{CONCLUSIONS}

In this work, we have shown in a combination of numerical modeling and analytical calculations that proximitized semiconducting carbon nanotubes are predestined hosts for Majorana bound states. While the numerical approach has allowed us to test the robustness of the topological phase against disorder or field misalignement, the analytical model has given us the possibility to clearly identify the phase boundaries of the topological phase transition, all issues crucial for an experimental observation. The use of semiconducting nanotubes instead of metallic ones as proposed in Refs. $[18,20,21]$ has the crucial advantage that - due to the much smaller Fermi velocity - the Majorana modes already emerge in CNTs of few micrometers in length, routinely accessible in experiments. In our setup, perpendicular magnetic fields of around $10 \mathrm{~T}$ are required to reach the topological phase. Thus, besides involving semiconducting CNTs, we propose the use of a thin layer of superconducting $\mathrm{NbSe}_{2}$ to induce the proximity effect. In fact, this material is known to sustain very large in plane magnetic fields before superconductivity is destroyed. With all experimental requirements being in the reach of state-of-the-art technology, we are confident that our work will stimulate experimental research to engineer Majorana modes in CNTs. Preliminary investigations in this direction have already been started [45].

\section{ACKNOWLEDGMENTS}

The authors thank the Deutsche Forschungsgemeinschaft for financial support via GRK 1570 and IGK "Topological insulators" grants, as well as the JSPS for the KAKENHI Grants (No. JP15K05118, No. JP15KK0147, and No. JP16H01046). We acknowledge the useful discussions with J. Klinovaja, M. Wimmer, and K. Flensberg. We are grateful to B. Siegert for his advice regarding the numerical calculations.

\section{APPENDIX A: VALLEY MIXING}

The possibility of tunneling between the two components of a normal-superconducting (N-S) hybrid system, which generates the pairing correlations in the normal part implies close contact between the $\mathrm{N}$ and $\mathrm{S}$ systems. With this close contact, the $\mathrm{N}$ wave functions are affected by the $\mathrm{S}$ substrate lattice potential, registering an increased electrostatic potential in the vicinity of the N-S contact area. In order to preserve the translational symmetry of the system, which allows us to construct Bloch bands of the bulk nanotube, we treat the electrostatic potential of the substrate as a continuous ridge, adding an on-site potential term to the Hamiltonian of the CNT at the atomic sites in the proximity of the superconducting substrate. We have tested several shapes of this ridge with similar values of the resulting valley mixing energy scale, $\Delta_{K K^{\prime}}$. For all calculations presented here, we chose a Gaussian form of $V(\varphi)$, shown in Fig. 10(a) and given by

$$
V(\varphi)=V_{0} \exp \left(-\left(\varphi-\varphi_{0}\right)^{2} / \Delta \varphi^{2}\right),
$$

where $\varphi$ is the angular coordinate of the nanotube atom, $V_{0}$ is an arbitrarily chosen maximum height of the substrate's potential, $\varphi_{0}$ is the shift between nanotube coordinates and the CNT-S contact line, and $\Delta \varphi$ controls the sharpness of the potential. The influence of the substrate potential for three different nanotube chiralities is illustrated in Fig. 10(b), where the atoms of the respective CNT's unit cell are colored according to the value of $V(\varphi)$ at this position. In the numerical calculations, we assumed $V_{0}=0.4 \mathrm{eV}, \varphi_{0}=90^{\circ}$, and $\Delta \varphi=2.5^{\circ}$.

We shall now assess the hybridization between different momentum states in the normal CNT, $|\boldsymbol{k}\rangle$ and $\left|\boldsymbol{k}^{\prime}\right\rangle$. First, we 
(a)

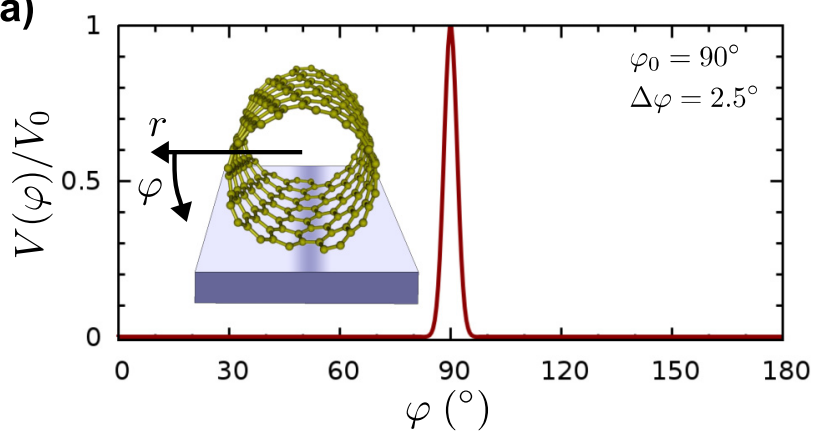

(b)

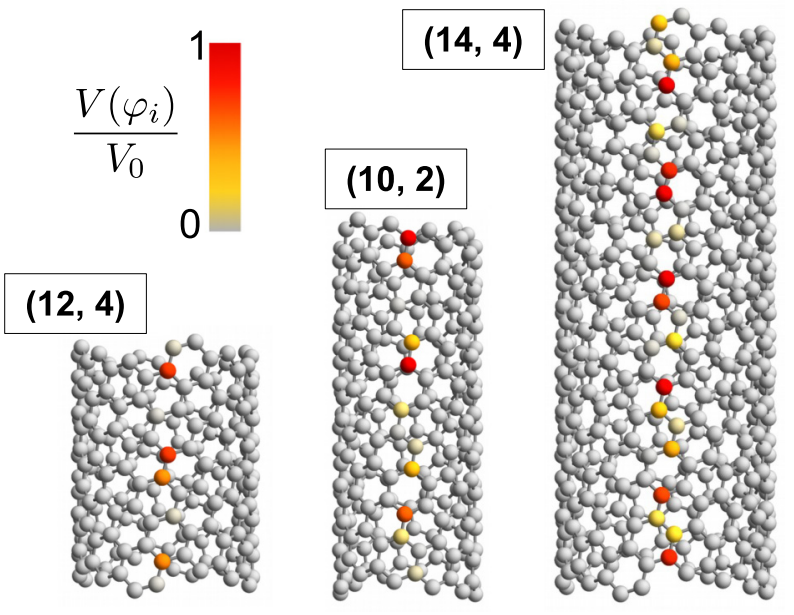

FIG. 10. (a) The substrate potential $V(\varphi)$ in the Gaussian form. The inset shows the nanotube coordinates in relation to the substrate. (b) Examples of unit cells of nanotubes with different chiralities, with the atoms along the contact area colored by their value of $V(\varphi)$.

introduce the basis of LCAO plane waves,

with

$$
|\boldsymbol{k}, p\rangle=\frac{1}{\sqrt{N_{c} N_{L}}} \sum_{\boldsymbol{R}} e^{i \boldsymbol{k} \cdot \boldsymbol{R}}|\boldsymbol{R}, p\rangle,
$$

$$
\langle\boldsymbol{r} \mid \boldsymbol{R}, p\rangle=p_{z}\left(\boldsymbol{r}-\left(\boldsymbol{R}+\delta \boldsymbol{R}_{p}\right)\right),
$$

where $N_{L}$ is the number of CNT unit cells and $N_{c}$ is the number of lattice sites (graphene's unit cells) in the CNT's unit cell. The index $p=A, B$ denotes the sublattice, $\boldsymbol{R}$ are the Bravais lattice vectors, and $\delta \boldsymbol{R}_{p}$ denote the shift of the $p$ atom from the center of graphene's unit cell. The CNT lattice and the relevant spatial quantities (coordinates, lattice vectors, atomic positions) are illustrated in Fig. 11. We approximate the wave functions of electronic $p_{z}$ orbitals by Dirac deltas.

This means that our $V(\varphi)$, which is diagonal in position and hence diagonal in sublattice, will only yield nonzero coupling between LCAO plane waves on the same sublattice,

$$
\begin{aligned}
V_{p}\left(\boldsymbol{k}, \boldsymbol{k}^{\prime}\right):= & \left\langle\boldsymbol{k}, p|V(\varphi)| \boldsymbol{k}^{\prime}, p\right\rangle \\
= & \frac{1}{N_{c} N_{L}} \sum_{\boldsymbol{R}} V\left(\varphi_{\boldsymbol{R}, p}\right) e^{i\left(\boldsymbol{k}-\boldsymbol{k}^{\prime}\right) \cdot \boldsymbol{R}} \\
= & \frac{1}{N_{L}} \sum_{n=1}^{N_{L}} e^{i\left(k-k^{\prime}\right) n a} \\
& \times \frac{1}{N_{c}} \sum_{j=1}^{N_{c}} V\left(\varphi_{p j}\right) e^{i\left(l_{\perp}-l_{\perp}^{\prime}\right) \varphi_{j}} e^{i\left(k-k^{\prime}\right) z_{j}},
\end{aligned}
$$

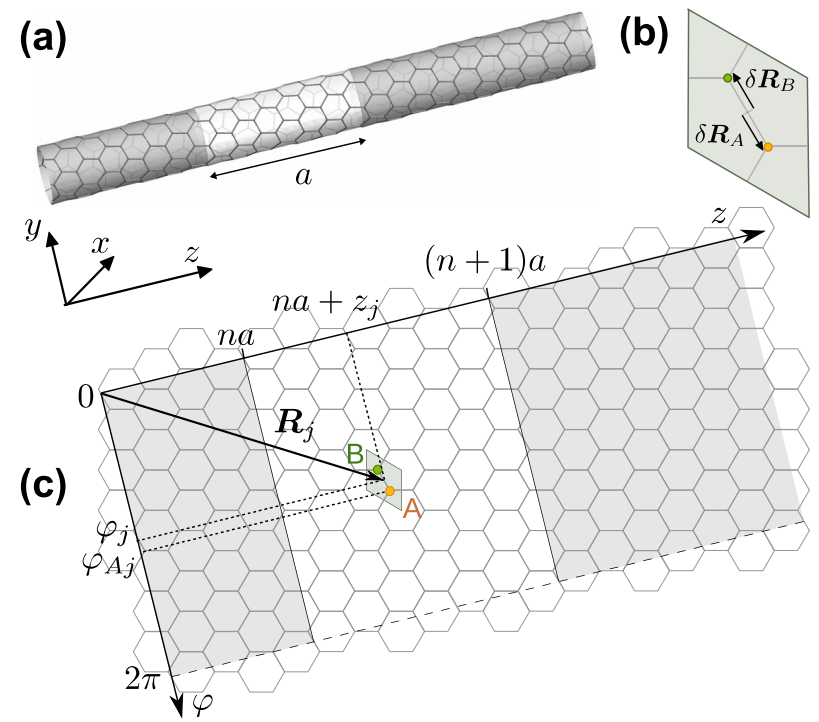

FIG. 11. (a) Fragment of a $(6,2)$ CNT lattice. The white area marks the translational unit cell of the CNT. (b) The unit cell of graphene, with the $A$ and $B$ sublattice atoms and their shifts $\delta \boldsymbol{R}_{A / B}$ from the center of the unit cell. (c) Unrolled nanotube lattice and the quantities used in Eqs. (A2) and (A3).

where in the last step we split the sum over lattice sites $\boldsymbol{R}$ into a sum over nanotube unit cells indexed by $n$ and a sum over all atoms in one unit cell, indexed by $j$. The lattice constant $a$ is the length of the CNT's unit cell, $\varphi_{j}$ is the angular coordinate of the lattice site $\boldsymbol{R}_{j}$, and $\varphi_{p j}$ the angular coordinate of the $p$ sublattice atom belonging to this site. The quantities $l_{\perp}, l_{\perp}^{\prime}$ are the angular momentum components of $\boldsymbol{k}$ and $\boldsymbol{k}^{\prime}$, respectively. The summation over the unit cells in an infinite CNT yields the selection rule for the longitudinal momentum, $k=k^{\prime}$, while the summation over lattice sites determines the strength with which different angular momentum states at the same $k$ are coupled. The angular momenta in the $K$ and $K^{\prime}$ valley have opposite signs, $l_{K}=-l_{K^{\prime}}$. When the $l_{K}$ appropriate for a given chirality is inserted into (A3), we obtain the $k$-independent coupling between LCAO plane waves from $K$ and $K^{\prime}$ valley,

$$
V_{K K^{\prime}, p}=\frac{1}{N_{c}} \sum_{j=1}^{N_{c}} V\left(\varphi_{p j}\right) e^{2 i l_{K} \varphi_{j}} .
$$

This quantity is in general complex, with different phases on the $A$ and $B$ sublattice, but with the same absolute value, $\left|V_{K K^{\prime}, A}\right|=\left|V_{K K^{\prime}, B}\right|=:\left|V_{K K^{\prime}}\right|$, shown in Fig. 12. The value of $\left|V_{K K^{\prime}}\right|$ is not sensitive to the precise placement of the CNT on the substrate, i.e., to the value of $\varphi_{0}$, provided the decay angle $\Delta \varphi$ is large enough $\left(>2-4^{\circ}\right)$.

Upon conversion to the conduction/valence band basis (i.e., the basis of the CNT Bloch states), we obtain the valley mixing $\Delta_{K K^{\prime}}(k)$, which is proportional to $\left|V_{K K^{\prime}}\right|$ but depends on the value of $k$. As a result, the minimum of the bands is displaced from the curvature-shifted Dirac points, as can be seen by comparing the colored and grey lines in Fig. 1(b). For the sake of simplicity in the effective model, we accommodate this displacement through a modification of the curvature shift $\Delta k_{\|}^{c}$, defined in Sec. III, and take the value of $\Delta_{K K^{\prime}}$ to be constant 


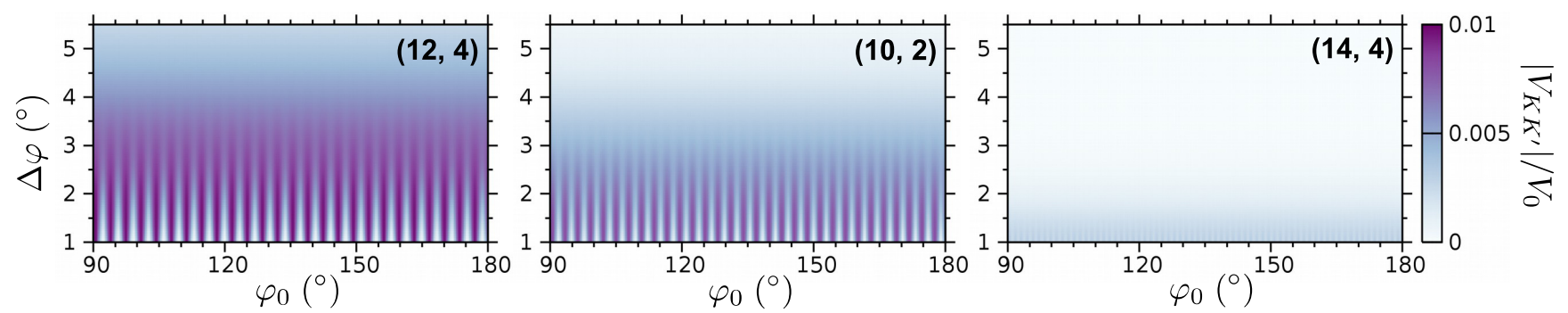

FIG. 12. Calculated plane-wave coupling $\left|V_{K K^{\prime}}\right|$ as a function of the substrate potential parameters $\varphi_{0}, \Delta \varphi$. It is most effective in nanotubes with shorter unit cells, and in all of them the dependence on $\varphi_{0}$ vanishes beyond some value of $\Delta \varphi$.

in $k$, fitted from the width of the $K / K^{\prime}$ anticrossing in the numerically obtained band structure. This simplification eases greatly the analytical calculation, while keeping the agreement between our numerical and effective model results, as the following sections will show.

\section{APPENDIX B: TOPOLOGICAL INVARIANTS}

The symmetries of the BdG Hamiltonian (10) can be expressed in terms of Pauli matrices, denoted by $\pi$ in the particle-hole (Nambu) subspace, by $\tau$ in the valley subspace, and by $s$ in the spin subspace. The particle-hole symmetry operator $\mathcal{P}$, such that $\mathcal{P} \mathcal{H}_{\mathrm{BdG}}(k) \mathcal{P}^{-1}=-\mathcal{H}_{\mathrm{BdG}}(-k)$, is given by $\mathcal{P}=\pi_{x} \otimes \tau_{x} \otimes s_{x} \mathcal{K}$, where $\tau_{0}$ and $s_{0}$ are the identities in their respective subspaces and $\mathcal{K}$ denotes the operator of the complex conjugation. The Hamiltonian $H_{\mathrm{BdG}}$ has also a chiral symmetry, i.e., it fulfills $C \mathcal{H}_{\mathrm{BdG}}(k) C^{-1}=-\mathcal{H}_{\mathrm{BdG}}(k)$ with a unitary operator $C$. The operator is given by $C=\pi_{y} \otimes \tau_{0} \otimes s_{0}$. The presence of those two symmetries implies that there exists a third one, which we call $\tilde{\mathcal{T}}=C \mathcal{P}^{-1}$ and which fulfills $\tilde{\mathcal{T}} \mathcal{H}_{\mathrm{BdG}}(k) \tilde{\mathcal{T}}^{-1}=\mathcal{H}_{\mathrm{BdG}}(-k)$. Its expression in this basis is $\tilde{\mathcal{T}}=-i \pi_{z} \otimes \tau_{x} \otimes s_{x} \mathcal{K}$. The operation $\tilde{\mathcal{T}}$ squares to +1 , hence it is clear that it is not the time reversal symmetry of a spin-1/2 system. The fact that it is diagonal in the Nambu space implies that already the nonsuperconducting Hamiltonian $H(k)(11)$ is invariant under a restricted $\tilde{\mathcal{T}}_{\text {red }}=\tau_{x} \otimes s_{x} \mathcal{K}$, which is indeed the case and reflects a physical symmetry of the system. It is the symmetry of rotation with respect to an axis perpendicular to the CNT, which exchanges both the valley, longitudinal momentum and spin. It also exchanges the sublattices, which accounts for its $\mathcal{K}$ component. If, and only if, the magnetic field is also applied perpendicular to the CNT axis, the nonsuperconducting Hamiltonian is invariant under $\tilde{\mathcal{T}}_{\text {red }}$.

Pfaffian $\left(\mathbb{Z}_{2}\right)$ invariant. In systems with particle-hole symmetry, the topological invariant $W$ can be evaluated using the representation of the Hamiltonian in the Majorana basis, i.e., the basis of eigenstates of $\mathcal{P}$ [36], obtained by a transformation $U_{M}, \mathcal{H}_{M}(k)=U_{\mathrm{M}} \mathcal{H}_{\mathrm{BdG}}(k) U_{\mathrm{M}}^{\dagger}$. We can define a matrix $X$ by $i X(k)=\mathcal{H}_{M}(k)$. At the time reversal invariant momenta $k=0, \pi / a, X(k)$ is a real and skew symmetric matrix, $X(k)=-[X(k)]^{T}$. The topological invariant $W$ can then be expressed through the Pfaffian of $X$ at $k=0, \pi / a$ [36], $W=\operatorname{sgn}\{\operatorname{Pf}[X(\pi / a)] \operatorname{Pf}[X(0)]\}= \pm 1$, which is of a $\mathbb{Z}_{2}$ type. For our system, the unitary matrix $U_{M}$ is given by

$$
U_{M}=\frac{1}{\sqrt{2}}\left(\begin{array}{cc}
\tau_{x} \otimes s_{x} & \tau_{0} \otimes s_{0} \\
-i \tau_{x} \otimes s_{x} & i \tau_{0} \otimes s_{0}
\end{array}\right)
$$

At time reversal invariant momenta $k=0, \pi / a, X(k)$ has the particularly simple form,

$$
X(k)=\left(\begin{array}{cc}
0 & H(k)+\Delta_{M} \\
-\left(H(k)+\Delta_{M}\right) & 0
\end{array}\right),
$$

where $\Delta_{M}=U_{M} \Delta U_{M}^{\dagger}$. Then the Pfaffian is calculated as $\operatorname{Pf}[X(k)]=\operatorname{det}\left[H(k)+\Delta_{M}\right]$. We calculated the topological phase diagrams with the $W$ invariant numerically, assuming $\operatorname{sgnPf}[X(\pi / a)]=+1$ thus checking only for the band inversion at $k=0$.

Winding number $(\mathbb{Z})$ invariant. Since the $\mathrm{BdG}$ Hamiltonian has the chiral symmetry $\left\{C, \mathcal{H}_{\mathrm{BdG}}\right\}=0$, one can introduce the winding number $v=-\frac{1}{4 \pi i} \int_{\mathrm{BZ}} \operatorname{Tr}\left[C \mathcal{H}_{\mathrm{BdG}}^{-1}(k) \partial_{k} \mathcal{H}_{\mathrm{BdG}}(k)\right]$ as a 1D topological invariant $[46,47]$. The identity with another definition of the winding number, which uses a flat band Hamiltonian [48], is proven in Appendix C1 in Ref. [40]. Let us consider the unitary transformation

$$
U_{c}=\frac{1}{2}\left(\begin{array}{cc}
1+i & 1+i \\
-1+i & 1-i
\end{array}\right) \otimes \tau_{0} \otimes s_{0},
$$

which rotates the Pauli matrices for the particle-hole basis as $U_{c}^{\dagger} \pi_{x} U_{c}=\pi_{y}, U_{c}^{\dagger} \pi_{y} U_{c}=\pi_{z}, U_{c}^{\dagger} \pi_{z} U_{c}=\pi_{x}$. Correspondingly, the Hamiltonian in Eq. (10) takes an off-diagonal form,

$$
\mathcal{H}_{c}(k)=U_{c}^{\dagger} \mathcal{H}_{\mathrm{BdG}}(k) U_{c}=\left(\begin{array}{cc}
0 & H(k)-i \Delta \\
(H(k)-i \Delta)^{\dagger} & 0
\end{array}\right) .
$$

Because the chiral operator is transformed as $C_{c}=$ $U_{c}^{\dagger} C U_{c}=\pi_{z}$, the winding number is written as $v=$ $\frac{1}{2 \pi} \int_{\mathrm{BZ}} d k \partial_{k} \arg \operatorname{det}(H(k)-i \Delta)$. The topological invariant $v_{l}$ for the band $l$ can be shown to be $\mathbb{Z} \ni \nu_{l}=2 \gamma_{l}$ if $\gamma_{l}$ is calculated in the basis of chiral symmetry eigenstates. Therefore $W=$ $\exp \left(i \pi \sum_{l} v_{l}\right)= \pm 1$.

\section{APPENDIX C: GAP CLOSING CONDITION}

In the basis in which the operator $C$ is diagonal, discussed above, the $\mathrm{BdG}$ Hamiltonian is given by

$$
\mathcal{H}_{\mathrm{BdG}}^{C}(k)=\left(\begin{array}{cc}
0 & D(k) \\
D^{\dagger}(k) & 0
\end{array}\right)
$$

where $D(k)=H(k)-i \Delta$. In order to obtain the gap closing condition, we square the $\mathrm{BdG}$ Hamiltonian in chiral basis, which yields

$$
\left(\mathcal{H}_{\mathrm{BdG}}^{C}(k)\right)^{2}=\left(\begin{array}{cc}
D(k) D^{\dagger}(k) & 0 \\
0 & D^{\dagger}(k) D(k)
\end{array}\right) .
$$


This matrix has zero-energy eigenvalues at the $\Gamma$ point if $\operatorname{det}\left(D(k=0) D^{\dagger}(k=0)\right)=\operatorname{det}\left(D^{\dagger}(k=0) D(k=0)\right)=0$. From this, we obtain the exact gap closing condition at the $\Gamma$ point, given by Eq. (14).

\section{APPENDIX D: TWO-BAND EFFECTIVE MODEL}

The four-band model, while approximating very well the numerical results, is rather intractable analytically. We can simplify it, using the knowledge of the energy scales in our system. The largest relevant energy scales are $\Delta_{\mathrm{SO}}$ and $\Delta_{K K^{\prime}}$, similar in magnitude, with $\Delta_{\mathrm{SO}}=2 \mathrm{meV}$ and $\Delta_{K K^{\prime}}=$ $2.5 \mathrm{meV}$. Two smaller energy scales are the superconducting gap $\Delta_{0}=0.4 \mathrm{meV}$, and the Zeeman energy $E_{Z}$. The latter can be tuned continuously, but near the boundaries of the nontrivial topological phase has similar magnitude as $\Delta_{0}$. Our strategy is therefore to diagonalize the initial single-particle Hamiltonian of the CNT (6) together with the valley-mixing term (7) exactly, express the Zeeman term (8) in this basis, and omit the terms coupling the lower and upper band pairs. The two resulting subspaces contain only one band pair each, halving the dimensions of the Hamiltonians under our treatment.

\section{Construction of the single-particle Hamiltonian}

The CNT Hamiltonian $H_{\mathrm{CNT}}-\mu N+H_{\Delta_{K K^{\prime}}}$ can be brought to a diagonal form by employing the unitary transformation

$$
\left(\begin{array}{c}
c_{k K s} \\
c_{k K^{\prime} s}
\end{array}\right)=\left(\begin{array}{cc}
a_{s}(k) & b_{s}(k) \\
-b_{s}^{\star}(k) & a_{s}^{\star}(k)
\end{array}\right)\left(\begin{array}{c}
\alpha_{k s} \\
\beta_{k s}
\end{array}\right),
$$

with $\left|a_{s}(k)\right|^{2}+\left|b_{s}(k)\right|^{2}=1$. It is diagonalized by the following values of $a_{s}(k)$ and $b_{s}(k)$ :

$$
\begin{aligned}
\left|a_{s}(k)\right|^{2} & =\frac{1}{2}\left(1-\frac{\xi_{K s}(k)-\xi_{K^{\prime} s}(k)}{\sqrt{\left(\xi_{K s}(k)-\xi_{K^{\prime} s}(k)\right)^{2}+4\left|\Delta_{K K^{\prime}}\right|^{2}}}\right), \\
\left|b_{s}(k)\right|^{2} & =\frac{1}{2}\left(1+\frac{\xi_{K s}(k)-\xi_{K^{\prime} s}(k)}{\sqrt{\left(\xi_{K s}(k)-\xi_{K^{\prime} s}(k s)\right)^{2}+4\left|\Delta_{K K^{\prime}}\right|^{2}}}\right),
\end{aligned}
$$

and $\arg \left(a_{s}(k)\right)=\arg \left(b_{s}(k)\right)=\frac{\phi}{2}$ with $\phi=\arg \left(\Delta_{K K^{\prime}}\right)$. With these we obtain

$$
H_{\mathrm{CNT}}-\mu N+H_{\Delta_{K K^{\prime}}}=\sum_{k, s} E_{+s} \beta_{k s}^{\dagger} \beta_{k s}+E_{-s} \alpha_{k s}^{\dagger} \alpha_{k s},
$$

where the eigenvalues are defined in the following way:

$$
\begin{aligned}
E_{ \pm s}(k)= & \frac{1}{2}\left(\xi_{K s}(k)+\xi_{K^{\prime} s}(k)\right) \\
& \pm \frac{1}{2} \sqrt{\left(\xi_{K s}(k)-\xi_{K^{\prime} s}(k)\right)^{2}+4\left|\Delta_{K K^{\prime}}\right|^{2}} .
\end{aligned}
$$

Due to the time-reversal conjugation of $\xi_{\tau s}(k)=\xi_{-\tau-s}(-k)$, it can be shown that $\left|a_{s}(k)\right|=\left|b_{-s}(-k)\right|$ and $E_{ \pm s}(k)=$ $E_{ \pm-s}(-k)$. These four eigenvalues are shown in Fig. 13(a).

Now we will express the Zeeman term (8) in this basis,

$$
\begin{aligned}
H_{Z}= & \sum_{k s} \mu_{B} \tilde{B}_{\perp}\left(\alpha_{k s}^{\dagger} \alpha_{k,-s}+\beta_{k s}^{\dagger} \beta_{k,-s}\right) \\
& +s \mu_{B} B_{\perp}^{\star}\left(\alpha_{k s}^{\dagger} \beta_{k,-s}-\beta_{k s}^{\dagger} \alpha_{k,-s}\right),
\end{aligned}
$$

where $\tilde{B}_{\perp}$ and $B_{\perp}^{\star}$ are the renormalized magnetic field components. Using Eqs. (D2), we can express them as

$$
\begin{aligned}
\tilde{B}_{\perp} & =B_{\perp}\left(\left|a_{\uparrow}(k)\right|\left|a_{\downarrow}(k)\right|+\left|b_{\uparrow}(k)\right|\left|b_{\downarrow}(k)\right|\right), \\
B_{\perp}^{\star} & =B_{\perp}\left(\left|a_{\uparrow}(k)\right|\left|b_{\downarrow}(k)\right|-\left|b_{\uparrow}(k)\right|\left|a_{\downarrow}(k)\right|\right) .
\end{aligned}
$$

The magnetic field $\tilde{B}_{\perp}$ couples the spins within the lower and upper band pair, while $B_{\perp}^{\star}$ couples the spins between band pairs. As long as the energy difference between the lower and upper band pairs is larger than the Zeeman energy, $\Delta E=\left|E_{+s}-E_{--s}\right|>\mu_{B} B_{\perp}$, we can omit the terms with $B_{\perp}^{\star}$. The upper and lower pairs of bands can now be treated separately. We shall proceed to find the solutions for the lower band pair only, assuming that the chemical potential $\mu$ is tuned into the gap between the two energy bands $\tilde{E}_{1}$ and $\tilde{E}_{2}$. Therefore we will neglect the influence of the bands $E_{3}$ and $E_{4}$ because those bands are not occupied. Similar calculation can be performed for the upper pair, neglecting the lower. The Hamiltonian for the two lowest energy bands is given by

$$
\tilde{H}_{-}=\sum_{k, s} E_{-s} \alpha_{k s}^{\dagger} \alpha_{k s}+\mu_{B} \tilde{B}_{\perp} \alpha_{k s}^{\dagger} \alpha_{k,-s} .
$$

This Hamiltonian can be diagonalized by the transformation

$$
\left(\begin{array}{c}
\alpha_{k \uparrow} \\
\alpha_{k \downarrow}
\end{array}\right)=\left(\begin{array}{cc}
s(k) & t(k) \\
-t(k) & s(k)
\end{array}\right)\left(\begin{array}{c}
f_{k 1} \\
f_{k 2}
\end{array}\right),
$$

where the coefficients must satisfy $s(k)^{2}+t(k)^{2}=1$. The new quantum number in (D6) $i \in\{1,2\}$ just reflects the ordering of the energy bands $E_{1}<E_{2}$. The coefficients $s(k)$ and $t(k)$ are defined as

$$
\begin{aligned}
s^{2}(k) & =\frac{1}{2}\left(1-\frac{E_{-\uparrow}(k)-E_{-\downarrow}(k)}{\sqrt{\left(E_{-\uparrow}(k)-E_{-\downarrow}(k)\right)^{2}+4\left(\mu_{B} \tilde{B}_{\perp}\right)^{2}}}\right), \\
t^{2}(k) & =\frac{1}{2}\left(1+\frac{E_{-\uparrow}(k)-E_{-\downarrow}(k)}{\sqrt{\left(E_{-\uparrow}(k)-E_{-\downarrow}(k)\right)^{2}+4\left(\mu_{B} \tilde{B}_{\perp}\right)^{2}}}\right) .
\end{aligned}
$$

The coefficients satisfy the following time-reversal conjugation $s(k)=t(-k)$. Then, the full Hamiltonian with decoupled band pairs in its diagonal basis is given by

$$
\tilde{H}_{\mathrm{CNT}}=\sum_{k} \sum_{i=1}^{2} \tilde{E}_{i} f_{k i}^{\dagger} f_{k i}
$$

with the corresponding single-particle energies

$$
\begin{aligned}
\tilde{E}_{i}(k)= & \frac{1}{2}\left(E_{-\uparrow}(k)+E_{-\downarrow}(k)\right) \\
& +(-1)^{i} \frac{1}{2} \sqrt{\left(E_{-\uparrow}(k)-E_{-\downarrow}(k)\right)^{2}+4\left(\mu_{B} \tilde{B}_{\perp}\right)^{2}} .
\end{aligned}
$$

The single-particle energies have the property $\tilde{E}_{i}(k)=\tilde{E}_{i}(-k)$ with $i \in\{1,2\}$ because $\tilde{B}_{\perp}(k)=\tilde{B}_{\perp}(-k)$. The renormalized magnetic field opens a band gap at the $\Gamma$ point. Figure 13(a) shows the four bands $\tilde{E}_{1 / 2 / 3 / 4}$ for magnetic field strengths $B_{\perp}=0,10,50 \mathrm{~T}$. At $B_{\perp}=10 \mathrm{~T}$, the energies obtained in the two-band model still agree very well with those of the full four-band model. 

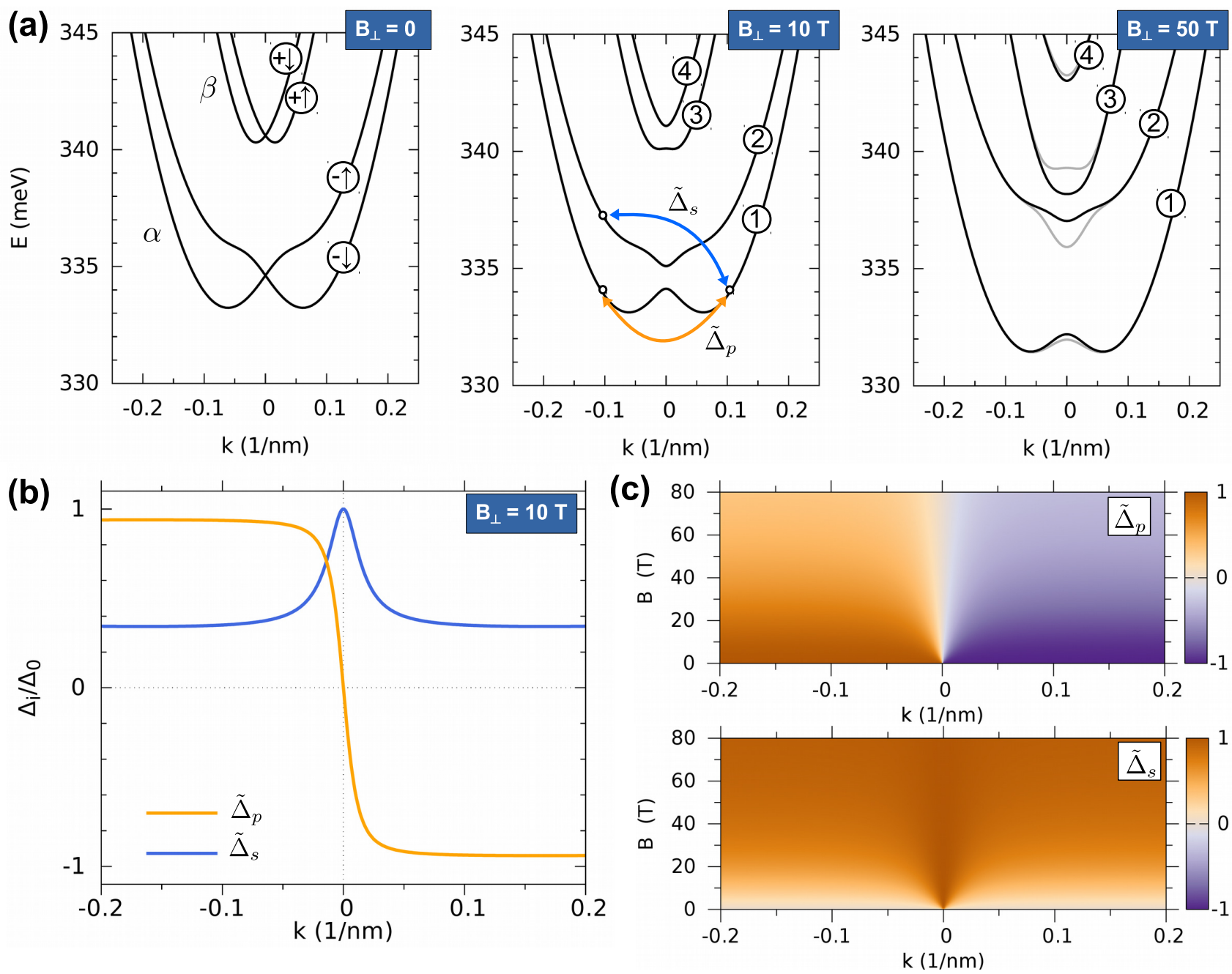

FIG. 13. (a) The two pairs of bands $\beta$ and $\alpha$ with black lines showing the energies $E_{ \pm s}$, respectively, at $B_{\perp}=0$, and $\tilde{E}_{1 / 2 / 3 / 4}$ at $B_{\perp}=10$ and $50 \mathrm{~T}$. The grey lines show the corresponding solutions of the four-band model. The approximation decoupling the upper pair from the lower holds at small fields, until $B_{\perp}^{*}$ becomes too large to be neglected and the two-band model becomes unreliable. Only the superconducting pairings in the same band pair are retained, with $\tilde{\Delta}_{p}$ acting within band and $\tilde{\Delta}_{s}$ pairing each member of the pair with its partner. (b) The dependence on $k$ of the two pairing terms at $B_{\perp}=10 \mathrm{~T}$, in $\Delta_{0}$ units. (c) The two pairing terms as functions of $k$ and $B_{\perp}$. Note that the amplitude of $\tilde{\Delta}_{s}$ at $k=0$ remains high throughout the whole $B_{\perp}$ range, unlike in the four-band model.

\section{Superconducting pairings}

Using the transformation (D1) the pairing Hamiltonian (9) becomes

$$
\begin{aligned}
H_{\mathrm{SC}}= & \sum_{k} \Delta_{+}(k)\left(\beta_{k \uparrow}^{\dagger} \beta_{-k \downarrow}^{\dagger}-\alpha_{k \uparrow}^{\dagger} \alpha_{-k \downarrow}^{\dagger}+\text { H.c. }\right) \\
& +\Delta_{-}(k)\left(\beta_{k \uparrow}^{\dagger} \alpha_{-k \downarrow}^{\dagger}+\alpha_{k \uparrow}^{\dagger} \beta_{-k \downarrow}^{\dagger}+\text { H.c. }\right),
\end{aligned}
$$

where we introduce the following definition:

$$
\begin{gathered}
\Delta_{+}(k)=\Delta_{0}\left(\left|a_{\uparrow}(k)\right|\left|b_{\downarrow}(-k)\right|+\left|b_{\uparrow}(k)\right|\left|a_{\downarrow}(-k)\right|\right), \\
\Delta_{-}(k)=\Delta_{0}\left(\left|a_{\uparrow}(k)\right|\left|a_{\downarrow}(-k)\right|-\left|b_{\uparrow}(k)\right|\left|b_{\downarrow}(-k)\right|\right) .
\end{gathered}
$$

For simplifications, we can use the time-reversal conjugation $\left|a_{s}(k)\right|=\left|b_{-s}(-k)\right|$ and by using the condition $\left|a_{s}(k)\right|^{2}+$ $\left|b_{s}(k)\right|^{2}=1$ we obtain that $\Delta_{+}(k)=\Delta_{0}$ and $\Delta_{-}(k)=0$, also if $\tilde{B}_{\perp} \neq 0$. Only $B_{\perp}^{\star} \neq 0$ would induce a finite $\Delta_{-}(k)$. Since we omit $B_{\perp}^{\star}, \Delta_{-}(k)$ is vanishing and we have again two separate pairings in the Hamiltonian. We can express the pairing Hamiltonian in the eigenbasis of the CNT with the transformation (D6) and by omitting the pairing with the upper bands we obtain

$$
\begin{aligned}
\tilde{H}_{\mathrm{SC}}= & \sum_{k} \Delta_{0}\left(s^{2}(k) f_{k 1}^{\dagger} f_{-k 1}^{\dagger}-t^{2}(k) f_{k 2}^{\dagger} f_{-k 2}^{\dagger}\right) \\
& +\Delta_{0} s(k) t(k)\left(f_{k 2}^{\dagger} f_{-k 1}^{\dagger}-f_{k 1}^{\dagger} f_{-k 2}^{\dagger}\right)+\text { H.c. }
\end{aligned}
$$

The Bogoliubov-de Gennes (BdG) Hamiltonian can be defined by $\tilde{H}_{12}=\frac{1}{2} \sum_{k} \Psi^{\dagger} \tilde{\mathcal{H}}_{\mathrm{BdG}} \Psi$ with the Nambu spinor $\Psi^{\dagger}=$ $\left(f_{k 1}^{\dagger}, f_{k 2}^{\dagger}, f_{-k 1}, f_{-k 2}\right)$. The corresponding BdG Hamiltonian for our system is given by

$$
\tilde{\mathcal{H}}_{\mathrm{BdG}}=\left(\begin{array}{cccc}
\tilde{E}_{1}(k) & 0 & \tilde{\Delta}_{p}(k) & -\tilde{\Delta}_{s}(k) \\
0 & \tilde{E}_{2}(k) & \tilde{\Delta}_{s}(k) & \tilde{\Delta}_{p}(k) \\
\tilde{\Delta}_{p}(k) & \tilde{\Delta}_{s}(k) & -\tilde{E}_{1}(k) & 0 \\
-\tilde{\Delta}_{s}(k) & \tilde{\Delta}_{p}(k) & 0 & -\tilde{E}_{2}(k)
\end{array}\right),
$$


with the pairing terms

$$
\begin{array}{r}
\tilde{\Delta}_{p}(k)=\Delta_{0}\left(s^{2}(k)-t^{2}(k)\right)=-\tilde{\Delta}_{p}(-k), \\
\tilde{\Delta}_{s}(k)=2 \Delta_{0} s(k) t(k)=\tilde{\Delta}_{s}(-k) .
\end{array}
$$

We see that the pairing term $\tilde{\Delta}_{s}(k)$ has an even and $\tilde{\Delta}_{p}(k)$ an odd parity, as shown in Fig. 13(b). We notice that these pairings depend on the magnetic field in a similar way as $\Delta_{s}(k)$ and $\Delta_{p}(k)$ in the four-band model, with differences visible only at high magnetic fields where the amplitude of $\tilde{\Delta}_{s}(k)$ around $k=0$ remains large, as can be seen in Fig. 13(c). From the conservation of energy it follows that $\tilde{\Delta}_{p}^{2}(k)+\tilde{\Delta}_{s}^{2}(k)=\Delta_{0}^{2}$.

Since $\tilde{\Delta}_{p}(k=0)=0$, the gap closing condition can be expressed directly as

$$
\tilde{\xi}_{ \pm}(k=0)=0
$$

where

$\tilde{\xi}_{ \pm}(k)=\frac{1}{2}\left(\tilde{E}_{1}(k)-\tilde{E}_{2}(k)\right) \pm \frac{1}{2} \sqrt{\left(\tilde{E}_{1}(k)+\tilde{E}_{2}(k)\right)^{2}+4 \tilde{\Delta}_{s}^{2}(k)}$.

This condition, neglecting the mixing between the band pairs, is shown with dashed lines in Figs. 4(b) and 4(d).

\section{APPENDIX E: INFLUENCE OF THE NEAREST-NEIGHBOR PAIRING $\Delta_{1}$}

The spin-singlet superconducting correlations can act both on-site and between nearest-neighbor sites [33]. When the nearest-neighbor pairing is stronger than the on-site pairing, $\Delta_{1}>\Delta_{0}$, a CNT can enter a nontrivial topological phase even in the absence of magnetic field [40], although the presence of time-reversal symmetry causes the zero-energy modes to be Dirac, rather than Majorana fermions [49]. We present here the topological phase diagrams obtained with the Pfaffian technique, for the range of $\Delta_{1} / \Delta_{0} \leqslant 10$. We keep the overall superconducting gap constant, $\sqrt{\Delta_{0}^{2}+\Delta_{1}^{2}}=0.4 \mathrm{meV}$.

In the basis of Bloch states, the pairing $\Delta_{1}$ becomes dependent on $k$ in a way similar to $\Delta_{K K^{\prime}}$ (cf. Appendix A), i.e., it becomes modulated by $\left|\sum_{j=1}^{3} \exp \left(i \boldsymbol{k} \cdot \Delta \boldsymbol{R}_{j}\right)\right|$, where $\Delta \boldsymbol{R}_{j}$ are lattice vectors between unit cells to which those nearest neighbors belong. That modulation for our $(12,4) \mathrm{CNT}$ is plotted in Fig. 14(a). The $\Delta_{0}$ term remains constant in the momentum space, with its relative strength of 1 also plotted for (a)

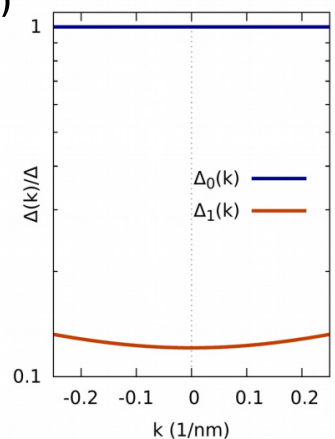

(c)

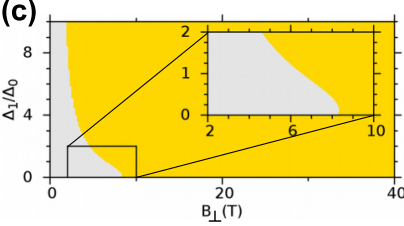

(b)

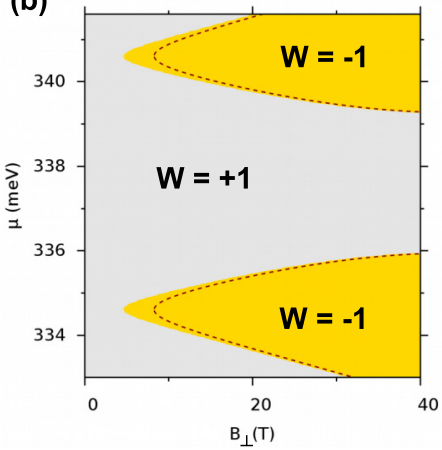

(d)

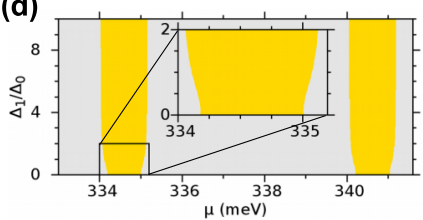

FIG. 14. (a) The $\Delta_{0}$ and $\Delta_{1}(k)$ pairing strength, in the units of the appropriate pairing type, bare $\Delta_{0}$ and bare $\Delta_{1}$, respectively. (b) Topological phase diagram constructed with the Pfaffian invariant, with bare $\Delta_{1}=2 \Delta_{0}$. Here and in (c) and (d) the value of $\sqrt{\Delta_{0}^{2}+\Delta_{1}^{2}}$ is kept constant and equal $0.4 \mathrm{meV}$. The red lines show the phase boundaries with $\Delta_{1}=0$. The inclusion of nearest-neighbor pairing has extended the nontrivial region towards lower magnetic field, but otherwise its influence is invisible. (c) Topological phase diagram at constant $\mu=334.6 \mathrm{meV}$. Here too the increasing contribution of $\Delta_{1}$ with respect to $\Delta_{0}$ extends the lower border of the nontrivial phase. (d) Topological phase diagram at $B_{\perp}=12 \mathrm{~T}$. Again, the inclusion of $\Delta_{1}$ slightly extends the borders of the nontrivial phase.

comparison. The topological phase diagram with $\Delta_{1}=2 \Delta_{0}$ is shown in Fig. 14(b). The nontrivial regions are extended farther towards low magnetic field, but at high $B_{\perp}$ the presence of $\Delta_{1}$ has no discernible influence.

The topological phase diagram in the $B_{\perp}, \Delta_{1} / \Delta_{0}$ plane at constant $\mu=334.6 \mathrm{meV}$ is shown in Fig. 14(c). Again, the visible variations occur only in the low-field ranges, and for low $\Delta_{1} / \Delta_{0}$ ratios. Beyond $\Delta_{1} / \Delta_{0} \approx 4$, the low-field boundary of the topological phase does not extend any further. Also when $B_{\perp}$ is kept constant, as shown in Fig. $14(\mathrm{~d})$ at $B_{\perp}=12 \mathrm{~T}$, the boundaries of nontrivial phase vary only slightly and mostly for $\Delta_{1} / \Delta_{0}<2$. In conclusion, the only relevant effect of $\Delta_{1}$ is that it allows the MQP to form at lower magnetic field, which is a bonus for experimentalists.

[1] E. Majorana, Teoria simmetrica dell'elettrone e del positrone, Il Nuovo Cimento (1924-1942) 14, 171 (1937).

[2] A. Yu. Kitaev, Unpaired Majorana fermions in quantum wires, Phys. Usp. 44, 131 (2001).

[3] R. M. Lutchyn, J. D. Sau, and S. Das Sarma, Majorana Fermions and a Topological Phase Transition in SemiconductorSuperconductor Heterostructures, Phys. Rev. Lett. 105, 077001 (2010).

[4] Y. Oreg, G. Refael, and F. von Oppen, Helical Liquids and Majorana Bound States in Quantum Wires, Phys. Rev. Lett. 105, 177002 (2010).
[5] V. Mourik, K. Zuo, S. M. Frolov, S. R. Plissard, E. P. A. M. Bakkers, and L. P. Kouwenhoven, Signatures of Majorana fermions in hybrid superconductor-semiconductor nanowire devices, Science 336, 1003 (2012).

[6] M. T. Deng, C. L. Yu, G. Y. Huang, M. Larsson, P. Caroff, and $\mathrm{H}$. Q. $\mathrm{Xu}$, Anomalous zero-bias conductance peak in a $\mathrm{Nb}-\mathrm{InSb}$ nanowire-Nb hybrid device, Nano Lett. 12, 6414 (2012).

[7] H. O. H. Churchill, V. Fatemi, K. Grove-Rasmussen, M. T. Deng, P. Caroff, H. Q. Xu, and C. M. Marcus, Superconductornanowire devices from tunneling to the multichannel regime: 
Zero-bias oscillations and magnetoconductance crossover, Phys. Rev. B 87, 241401 (2013).

[8] M. T. Deng, S. Vaitiekenas, E. B. Hansen, J. Danon, M. Leijnse, K. Flensberg, J. Nygård, P. Krogstrup, and C. M. Marcus, Majorana bound state in a coupled quantum-dot hybrid-nanowire system, Science 354, 1557 (2016).

[9] C.-X. Liu, J. D. Sau, T. D. Stanescu, and S. Das Sarma, Andreev bound states versus Majorana bound states in quantum dot-nanowire-superconductor hybrid structures: Trivial versus topological zero-bias conductance peaks, Phys. Rev. B 96, 075161 (2017).

[10] E. J. H. Lee, X. Jiang, R. Aguado, G. Katsaros, C. M. Lieber, and S. De Franceschi, Zero-Bias Anomaly in a Nanowire Quantum Dot Coupled to Superconductors, Phys. Rev. Lett. 109, 186802 (2012).

[11] T. D. Stanescu and S. Tewari, Majorana fermions in semiconductor nanowires: Fundamentals, modeling, and experiment, J. Phys.: Condens. Matter 25, 233201 (2013).

[12] S. Nadj-Perge, I. K. Drozdov, J. Li, H. Chen, S. Jeon, J. Seo, A. H. MacDonald, B. A. Bernevig, and A. Yazdani, Observation of Majorana fermions in ferromagnetic atomic chains on a superconductor, Science 346, 602 (2014).

[13] M. Ruby, F. Pientka, Y. Peng, F. von Oppen, B. W. Heinrich, and K. J. Franke, End States and Subgap Structure in ProximityCoupled Chains of Magnetic Adatoms, Phys. Rev. Lett. 115, 197204 (2015).

[14] Y. Peng, F. Pientka, L. I. Glazman, and F. von Oppen, Strong Localization of Majorana End States in Chains of Magnetic Adatoms, Phys. Rev. Lett. 114, 106801 (2015).

[15] R. Pawlak, M. Kisiel, J. Klinovaja, T. Meier, S. Kawai, T. Glatzel, D. Loss, and E. Meyer, Probing atomic structure and Majorana wavefunctions in mono-atomic Fe chains on superconducting $\mathrm{Pb}$ surface, Quantum Inf. 2, 16035 (2016).

[16] R. Saito, G. Dresselhaus, and M. S. Dresselhaus, Physical Properties of Carbon Nanotubes (Imperial College Press, London, 1998).

[17] E. A. Laird, F. Kuemmeth, G. A. Steele, K. Grove-Rasmussen, J. Nygård, K. Flensberg, and L. P. Kouwenhoven, Quantum transport in carbon nanotubes, Rev. Mod. Phys. 87, 703 (2015).

[18] R. Egger and K. Flensberg, Emerging Dirac and Majorana fermions for carbon nanotubes with proximity-induced pairing and spiral magnetic field, Phys. Rev. B 85, 235462 (2012).

[19] T. Kontos (private communication).

[20] J. Klinovaja, S. Gangadharaiah, and D. Loss, Electric-FieldInduced Majorana Fermions in Armchair Carbon Nanotubes, Phys. Rev. Lett. 108, 196804 (2012).

[21] J. D. Sau and S. Tewari, Topological superconducting state and Majorana fermions in carbon nanotubes, Phys. Rev. B 88, 054503 (2013).

[22] T. Ando, Spin-orbit interaction in carbon nanotubes, J. Phys. Soc. Jpn. 69, 1757 (2000).

[23] M. del Valle, M. Margańska, and M. Grifoni, Signatures of spin-orbit interaction in transport properties of finite carbon nanotubes in a parallel magnetic field, Phys. Rev. B 84, 165427 (2011).

[24] D. Tománek and S. G. Louie, First-principles calculation of highly asymmetric structure in scanning-tunneling-microscopy images of graphite, Phys. Rev. B 37, 8327 (1988).

[25] S. H. Jhang, M. Marganska, Y. Skourski, D. Preusche, B. Witkamp, M. Grifoni, H. van der Zant, J. Wosnitza, and C.
Strunk, Spin-orbit interaction in chiral carbon nanotubes probed in pulsed magnetic fields, Phys. Rev. B 82, 041404 (2010).

[26] F. Kuemmeth, S. Ilani, D. C. Ralph, and P. L. McEuen, Coupling of spin and orbital motion of electrons in carbon nanotubes, Nature 452, 448 (2008).

[27] W. Izumida, K. Sato, and R. Saito, Spin-orbit interaction in single wall carbon nanotubes: Symmetry adapted tight-binding calculation and effective model analysis, J. Phys. Soc. Jpn. 78, 074707 (2009).

[28] J. Klinovaja, M. J. Schmidt, B. Braunecker, and D. Loss, Carbon nanotubes in electric and magnetic fields, Phys. Rev. B 84, 085452 (2011).

[29] G. A. Steele, F. Pei, E. A. Laird, J. M. Jol, H. B. Meerwaldt, and L. P. Kouwenhoven, Large spin-orbit coupling in carbon nanotubes, Nat. Commun. 4, 1573 (2013).

[30] M. Marganska, P. Chudzinski, and M. Grifoni, The two classes of low energy spectra in finite carbon nanotubes, Phys. Rev. B 92, 075433 (2015).

[31] W. Izumida, R. Okuyama, A. Yamakage, and R. Saito, Angular momentum and topology in semiconducting single-wall carbon nanotubes, Phys. Rev. B 93, 195442 (2016).

[32] X. Xi, Z. Wang, W.Zhao, J.-H. Park, K. T. Law, H. Berger, László Forró, J. Shan, and K. F. Mak, Ising pairing in superconducting $\mathrm{NbSe}_{2}$ atomic layers, Nat. Phys. 12, 139 (2016).

[33] B. Uchoa and A. H. C. Neto, Superconducting States of Pure and Doped Graphene, Phys. Rev. Lett. 98, 146801 (2007).

[34] J. Gramich, A. Baumgartner, and C. Schönenberger, Subgap resonant quasiparticle transport in normal-superconductor quantum dot devices, Appl. Phys. Lett. 108, 172604 (2016).

[35] A direct sum $\boldsymbol{A} \oplus \boldsymbol{B}$ of a $p$-component vector $\boldsymbol{A}$ and an $q$ component vector $\boldsymbol{B}$ is a $p+q$-dimensional vector whose first $p$ components are those of $\boldsymbol{A}$ and the last $q$ are those of $\boldsymbol{B}$. Our $\Psi$ and $\chi^{n}$ are both $4 N$-dimensional vectors. The components of $\Psi$ are operators, while those of $\chi^{n}$ are complex numbers.

[36] C.-K. Chiu, J. C. Y. Teo, A. P. Schnyder, and S. Ryu, Classification of topological quantum matter with symmetries, Rev. Mod. Phys. 88, 035005 (2016).

[37] M. Sato and Y. Ando, Topological superconductors: A review, Rep. Prog. Phys. 80, 076501 (2017).

[38] R. M. Lutchyn and M. P. A. Fisher, Interacting topological phases in multiband nanowires, Phys. Rev. B 84, 214528 (2011).

[39] J. S. Lim, L. Serra, R. López, and R. Aguado, Magnetic-field instability of Majorana modes in multiband semiconductor wires, Phys. Rev. B 86, 121103 (2012).

[40] W. Izumida, L. Milz, M. Marganska, and M. Grifoni, Topology and zero energy edge states in carbon nanotubes with superconducting pairing, Phys. Rev. B 96, 125414 (2017).

[41] J. Cao, Q. Wang, and H. Dai, Electron transport in very clean, asgrown suspended carbon nanotubes, Nat. Mater. 4, 745 (2005).

[42] L.-M. Peng, Z. Zhang, S. Wang, and X. Liang, A doping-free approach to carbon nanotube electronics and optoelectronics, AIP Adv. 2, 041403 (2012).

[43] H. Ajiki and T. Ando, Electronic states in carbon nanotubes, J. Phys. Soc. Jpn. 62, 1255 (1993).

[44] C. L. Huang, J.-Y. Lin, Y. T. Chang, C. P. Sun, H. Y. Shen, C. C. Chou, H. Berger, T. K. Lee, and H. D. Yang, Experimental evidence for a two-gap structure of superconducting $\mathrm{NbSe}_{2}$ : A specific-heat study in external magnetic fields, Phys. Rev. B 76, 212504 (2007). 
[45] C. Strunk (private communication).

[46] X.G. Wen and A. Zee, Winding number, family index theorem, and electron hopping in a magnetic field, Nucl. Phys. B 316, 641 (1989).

[47] M. Sato, Y. Tanaka, K. Yada, and T. Yokoyama, Topology of Andreev bound states with flat dispersion, Phys. Rev. B 83, 224511 (2011).
[48] A. P. Schnyder, S. Ryu, A. Furusaki, and A. W. W. Ludwig, Classification of topological insulators and superconductors in three spatial dimensions, Phys. Rev. B 78, 195125 (2008).

[49] A. Haim, E. Berg, K. Flensberg, and Y. Oreg, No-go theorem for a time-reversal invariant topological phase in noninteracting systems coupled to conventional superconductors, Phys. Rev. B 94, 161110 (2016) 